\title{
Bathymetry-aided underwater acoustic localization using a single passive receiver
}

Elizaveta Dubrovinskaya, Paolo Casari, and Roee Diamant

Citation: The Journal of the Acoustical Society of America 146, 4774 (2019); doi: 10.1121/1.5138605

View online: https://doi.org/10.1121/1.5138605

View Table of Contents: https://asa.scitation.org/toc/jas/146/6

Published by the Acoustical Society of America

\section{ARTICLES YOU MAY BE INTERESTED IN}

Convolutional neural network for single-sensor acoustic localization of a transiting broadband source in very shallow water

The Journal of the Acoustical Society of America 146, 4687 (2019); https://doi.org/10.1121/1.5138594

Long-range frequency-difference source localization in the Philippine Sea

The Journal of the Acoustical Society of America 146, 4727 (2019); https://doi.org/10.1121/1.5138124

Source localization in range-dependent and time-varying shallow water: The Shallow Water 2006 experimental results

The Journal of the Acoustical Society of America 146, 4740 (2019); https://doi.org/10.1121/1.5138595

Automated localization of whales in coastal fjords

The Journal of the Acoustical Society of America 146, 4672 (2019); https://doi.org/10.1121/1.5138125

Introduction to the Special Issue on Acoustic Source Localization

The Journal of the Acoustical Society of America 146, 4647 (2019); https://doi.org/10.1121/1.5140997

A deep neural network approach to acoustic source localization in a shallow water tank experiment The Journal of the Acoustical Society of America 146, 4802 (2019); https://doi.org/10.1121/1.5138596

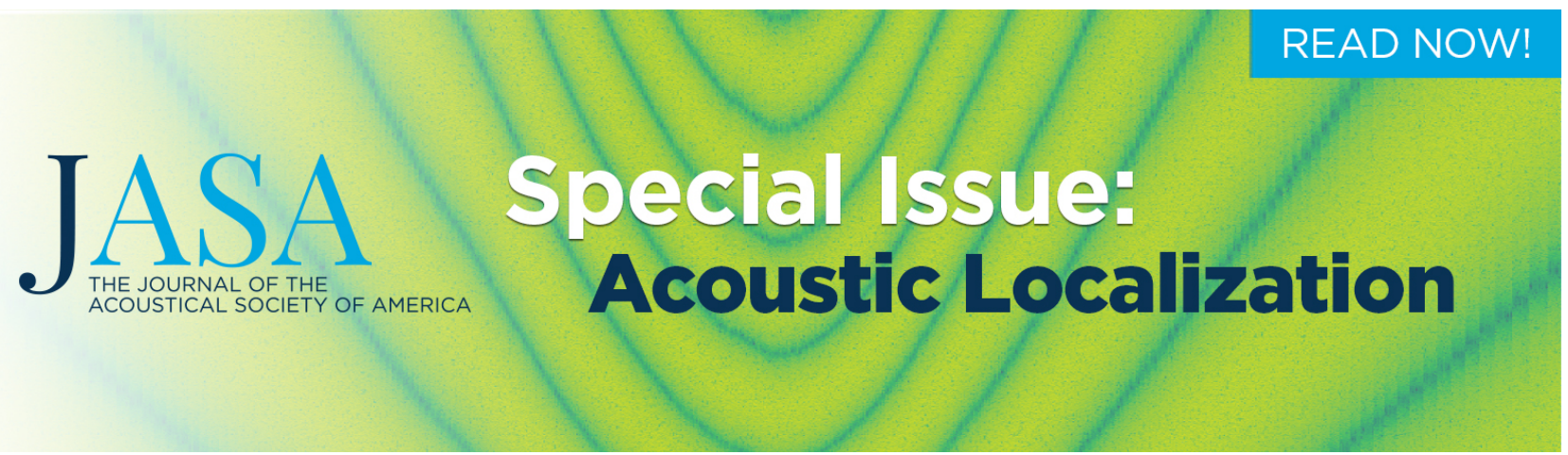




\title{
Bathymetry-aided underwater acoustic localization using a single passive receiver ${ }^{\text {a) }}$
}

\author{
Elizaveta Dubrovinskaya, ${ }^{1, \mathrm{~b})}$ Paolo Casari, ${ }^{1, \mathrm{c})}$ and Roee Diamant ${ }^{2}$ \\ ${ }^{1}$ Instituto Madrileño De Estudios Avanzados (IMDEA) Networks Institute, Madrid, Spain \\ ${ }^{2}$ Department of Marine Technologies, University of Haifa, Haifa, Israel
}

(Received 11 February 2019; revised 7 June 2019; accepted 28 June 2019; published online 31 December 2019)

\begin{abstract}
This paper considers the problem of estimating the trajectory of an autonomous underwater vehicle (AUV) via a single passive receiver, without any anchor nodes or receiving arrays, and with the only help of a sequence of known acoustic signals emitted by the AUV. This scenario is of interest in case multilateration-based alternatives would require the deployment of many receivers and imply exceedingly high costs, e.g., for the coverage of wide areas. The proposed method exploits the knowledge of environmental parameters such as the sound speed profile, bathymetry and bottom sediments in order to estimate the location of the AUV, taking advantage of the spatial dependency of channel impulse responses that arises from the diverse bathymetry around the receiver. This dependency is captured by comparing channel estimates against a database of channel responses, pre-computed through an acoustic propagation model. This yields multiple likely AUV locations, which are filtered via a path tracking method similar to the Viterbi algorithm, in order to estimate the trajectory of the AUV. Results obtained both from simulations and from a sea experiment show that the proposed method can estimate node locations and paths with a small error, especially considering the use of a single receiver. (C) 2019 Acoustical Society of America. https://doi.org/10.1121/1.5138605
\end{abstract}

$[\mathrm{KTW}]$

Pages: $4774-4789$

\section{INTRODUCTION}

Estimating the location of an autonomous underwater vehicle (AUV) is a required step for the operation of these devices for applications like ocean exploration, control of secure areas, and environmental monitoring. In these applications, the AUV covers large areas, and its self-navigation system may drift significantly. Localizing the AUV via noninertial systems may greatly help reduce such drift and improve the AUV's location reckoning. Localization is typically achieved through a set of fixed receiving hydrophones spread across the AUV deployment area. Yet, due to the wide area covered by the AUV during its mission, its transmissions tend to be detected very sparsely over both space and time. This is especially the case if the AUV's mission area is very large, and would imply the (expensive) deployment of a significant amount of equipment in order to cover the intended area with a sufficient density to enable reliable multilateration estimates. Instead, in order to balance a reasonable target detection probability with long term deployment constraints and costs, the coverage of large areas is typically achieved through sparse deployments. As a result, it is often the case that the signals used to detect a target are practically received only by a single receiver. Most existing algorithms to localize submerged devices require the presence of several anchor nodes, ${ }^{2}$ or prescribe message exchanges between the device and the

\footnotetext{
a) A preliminary version of this work has been presented at the IEEE WPNC 2017 Conference, Bremen, Germany.

b) Also at University Carlos III, Madrid, Spain.

c)Electronic mail: paolo.casari@imdea.org
}

anchors. ${ }^{3}$ Alternatively, range estimates from a single mobile anchor have been suggested assuming knowledge of the receiver's movement between subsequent transmissions through, e.g., acceleration measurements. ${ }^{4}$ Yet, this also requires interaction with the device to be localized.

In this paper, we propose a solution for the challenge of localizing a non-collaborative single AUV. As opposed to localization methods that rely on a receiving array, our method assumes only the presence of a single stationary and passive receiving element, and the knowledge of the transmitted signal (for example, the structure of the AUV pinger's signals), but does not require knowledge of the pinger's transmission times. Our approach is inspired by localization algorithms based on fingerprinting: ${ }^{5}$ these algorithms evaluate the correlation between some significant and distinguishable channel characteristics (e.g., the power-delay profile, the number of distinguishable arrivals, the angular spectrum of these arrivals, and so forth), and the same characteristics preliminarily measured at a number of locations and collected together in a fingerprint database. Instead, our method hinges on the spatial diversity of the sea bottom bathymetry to match the measured channel impulse response (CIR) with a set of CIRs generated through an acoustic propagation model. To that end, we target those environments where the bathymetry and the sound speed profile (SSP) in the water column induce different CIRs for different emitter-receiver location pairs. This is often the case for shallow-water environments with a diverse non-flat bathymetry, but also for deeper waters where sea bottom hills, mountains, or steep slopes may exist. 
We base our method on the modeling of expected acoustic CIRs for different possible locations of an acoustic source around the moored receiver. After measuring the CIR for each received signal, we correlate it with the pre-computed modeled CIRs in order to estimate the distance, depth, and bearing of the transmitter. This makes it possible to point the location of the sound-emitting AUV to the position for which the modeled CIR best fits the measured CIR. We repeat the process as the AUV moves and keeps emitting signals. The result is a sequence of location estimates whose size equals the number of detected sound emissions. These location estimates are expected to be noisy since there may be several modeled CIRs that are significantly correlated with each measured CIR. To filter this noise, we create a trellis of possible locations which are chosen from the output of the cross-correlation between the modeled and measured CIRs, and which satisfy a given maximum AUV speed. The final path of the AUV is obtained via an efficient trellis search process similar to the Viterbi algorithm.

Our contribution is twofold:

- A localization approach for an AUV using a single receiving element;

- An efficient method to reduce the state space resulting from the cross-correlation of modeled and measured CIRs, and thereby significantly decrease the complexity of the AUV path estimation process.

We evaluate our method through both simulations (based on real bathymetry and sound speed information) and a proof-of-concept sea trial. Our results show that the proposed approach can estimate the AUV path with an acceptable localization error.

The remainder of this paper is organized as follows: Section II provides an account of related work; Sec. III details the localization algorithm; Sec. IV presents simulation results; Sec. V describes our proof-of-concept sea trial; and Sec. VI concludes the paper.

\section{RELATED WORK}

\section{A. Techniques for underwater acoustic localization}

A comprehensive survey of underwater acoustic localization is presented in Refs. 6 and 7, and involves techniques for range estimation, bearing estimation, or both. Typical approaches to localization include long baseline ${ }^{7}$ (based on trilateration, and thus requiring the interaction between the device to be localized and the anchors), short baseline (usually operated from a single vessel), and ultra-short baseline systems, ${ }^{8}$ that estimate the location of the device via time of arrival (ToA) and angle of arrival measurements. As the accuracy of the angle estimation process directly depends on the stability of the equipment and is sensitive to a strong multipath, range-based approaches are more typically used.

Typical underwater ranging schemes rely on ToA, time difference of arrival, or received signal strength, which is translated into distance via an acoustic propagation model. ${ }^{9}$ ToA measurements can be obtained by separately analyzing the reflection patterns of transmitted signals, ${ }^{10}$ which can be estimated via matched filtering or by using phase-only correlation and the kurtosis metric to mitigate channelenhanced noise. ${ }^{11}$ Still, ToA measurements tend to be noisy due to multipath: mistaking a non-specular multipath component for the direct path is often regarded as measurement noise, ${ }^{12}$ and can be mitigated by transmitting signals having a narrow auto-correlation, ${ }^{13,14}$ or by averaging ToA measurements over different signals. ${ }^{15}$ Yet, instead of considering multipath as a distortion, the wealth of multipath arrivals can be exploited in passive systems in order to improve the localization accuracy, as well as to find the range of the acoustic source ${ }^{16}$ or to localize it with multiple receivers through a propagation model. ${ }^{17}$

In the literature, the closest approaches to our proposed scheme target localization with less than three reference nodes, often by exploiting some form of knowledge about the environment. For example, the work in Ref. 18 introduced a model-based range-bearing localization scheme that employs two receiving hydrophones. The method identifies multipath arrivals at the hydrophones and tracks them using a particle filter. An ambiguity surface is then constructed based on the expected multipath structure (derived via a ray model) and used to determine the most likely target location. To localize a source, the work in Ref. 5 proposes to match received signals against a set of fingerprints measured by an array of receivers. The authors test the feasibility of their approach in a pool, which represents a static environment where fingerprints remain sufficiently stable over time. However, systematic fingerprint measurements in uncontrolled open sea environments would be more challenging, due to the rapidly changing nature of underwater acoustic channels.

Matched-field processing, a family of array processingbased methods to estimate the parameters of the ocean waveguide based on the full field structure of acoustic signals, can also be extended to underwater localization. ${ }^{19}$ For example, the work in Ref. 20 assumes the three-dimensional knowledge of the SSP and of the bathymetry over a $600 \times 600 \mathrm{~km}^{2}$ area. The area is further divided into squares of side $5 \mathrm{~km}$ and normal mode theory is employed to predict sound propagation for a hypothetical source located in the center of each square. The sound field replicas thus obtained are matched to the acoustic field measurements collected through a 21element vertical array in order to infer the most likely location of the source. Matched-field localization has been recently achieved using compressed sensing (CS), which has the advantage of providing sparse solutions to inference problems using convex optimization. ${ }^{21}$ Specifically, the proposed approach employs CS (implemented through the basis pursuit algorithm and the Lasso path) to find the best match between field replicas and measurements, and shows that CS reliably handles coherent sources as well. Earlier, CS was considered to localize an underwater device by means of ultra-wideband radio CIR fingerprinting. ${ }^{22}$ Here, CS is implemented using the orthogonal matching pursuit and Lasso-II algorithms. Although the method achieves good localization accuracy, it remains suitable only for very short ranges due to the strong attenuation of radio frequency waves in salted waters. An approach to estimate the range of a source with respect to a single receiver is presented in Ref. 23. The 
authors assume that a moving source transmits signals with a period $\Delta t$ while moving around a hydrophone, and determine striation patterns in the function relating the signal observation time to $\Delta t$. These patterns are then employed to infer the velocity and range of the source based on the assumption that the ranging operations take place in a shallow-water environment with waveguide invariant $\beta=1$. When $\beta$ is unknown, the Automatic Identification System of nearby vessels can be opportunistically used to estimate it by relating their received signal, intensity, and frequency to their known position. ${ }^{24}$

The presence of an array of transmitters is assumed in Ref. 25, where the authors pre-compute the CIR from each transmitter at all points of a grid that finely covers the water column along a given bearing. The location of a receiver is estimated by comparing the CIRs measured by the receiver against pre-computed CIRs. The system finally employs the determined location to tune transmit beamforming. In Ref. 26, an AUV is located by fusing AUV heading and velocity information from some external sensors with acoustic phase information. The phase is measured from a batch of signals transmitted by a fixed projector of known location and received by a single hydrophone at the AUV.

\section{B. Differences with respect to indoor localization}

While fingerprinting is an established localization technique for terrestrial radio networks, ${ }^{27,28}$ one of its key assumptions is that radio measurements are repeatable and slowly varying in space $^{29}$ so that a device can actually afford to compute several statistics of a received radio signal and fuse them into a fingerprint vector. ${ }^{30}$ Conversely, the underwater acoustic channel tends to be much more dynamic, with several arrivals coming from multiple reflections over the surface, bottom, and volume scatterers. Moreover, the spatial coherence of the underwater channel is very limited, and a transmitter could experience very different channels when communicating to a static receiver from different locations. Similar uses of ray tracing to aid indoor localization (e.g., see Ref. 31) typically do not experience these issue, as they can rely on more stable radio channels. Filtering multiple sequential measurements through the Viterbi algorithm ${ }^{32}$ or other techniques (such as probability maps reproducing the expected movement of mobile devices ${ }^{33}$ or conditional random fields ${ }^{34}$ ) makes it possible to eliminate this uncertainty. However, the number of possible indoor positions to be matched by a terrestrial radio fingerprinting algorithm is usually limited, yielding a state space of tractable size. On the contrary, in our underwater approach the location of the target could be anywhere around the location of the single receiver, yielding an order-of $-10^{7}$ state space size. This calls for methods to reduce the complexity of trellis exploration. We also remark that direction-of-arrival fingerprinting-based localization has been reconsidered in the field of millimeter wave communications (e.g., see Refs. 35 and 36), where, however, the devices can leverage large arrays to reliably decouple propagation paths in the received angular spectra. This is in contrast with our assumption of using a single receiving element, and remains very different from the rich CIRs usually measured in underwater communications.

\section{Summary}

The literature that most closely relates to our paper is summarized in Table I, where we report the requirements, description, and shortcomings of each approach. From this comparison, it becomes clear that the most prominent contribution of our approach is the localization of a moving AUV in three-dimensions using a single receiver (and assuming only a single transmitter at the AUV). While our approach has some aspects in common with matched field processing and fingerprinting, it remains unique in that it reduces the ambiguity of the matching between measured and precomputed CIRs through a trellis search approach similar to the Viterbi algorithm, rather than resorting to fusing information from multiple transmitters or receivers. Moreover, we only process acoustic data, and do not require any external sensors to support the localization process.

With respect to the preliminary work in Ref. 1, the algorithm presented in this paper is much less sensitive to trellises that are not fully connected and to imperfect estimates of the initial AUV location; in addition, we include a performance verification through a sea experiment, and compare against benchmark approaches both in the simulations and in the sea experiment.

\section{ALGORITHM DESCRIPTION}

\section{A. Key idea}

We summarize the key idea behind our algorithm with the help of the flow chart in Fig. 1. We operate the AUV localization algorithm from a single receiver deployed at a known and well-explored stationary location. We assume that the sea bottom is diverse around the receiver (e.g., see Fig. 2), leading to a spatially-dependent CIR, which we exploit in order to estimate the location of the AUV via a fingerprinting-based location system. Since such a system requires to measure a three-dimensional database of fingerprints (which is not feasible in underwater scenarios due to the resource- and time-intensiveness of underwater acoustic measurements), we resort to a database of modeled CIRs instead. Such database is pre-computed via a numerical sound propagation model, such as the Bellhop ray tracing simulator (see Chap. 3 in Ref. 37, and Ref. 38).

Whenever an acoustic signal is received from the AUV, we estimate the CIR of the corresponding acoustic channel and correlate it with our database. In order to reduce the complexity of this step, we first correlate the CIR with specular and surface-reflected arrivals from the modeled CIRs: this excludes bearing-dependent bottom arrivals and allows us to retrieve a set of possible values for the AUV's depth and distance. We then compute one further round of crosscorrelations, this time with the whole CIR (thus including bottom reflections), for the selected depths and distances, and for every bearing value. The result is a number of possible AUV locations. We repeat the process for several subsequent acoustic signals emitted from the AUV, which may 
TABLE I. Summary of the most relevant related work.

\begin{tabular}{|c|c|c|c|c|}
\hline Ref. & Approach & Requirements & Details & Shortcomings \\
\hline $18(2015)$ & Multipath tracking & $\begin{array}{l}\text { - Two receivers } \\
\text { - Known environmental parameters }\end{array}$ & $\begin{array}{l}\text { - Compare multipath vs ray model } \\
\text { - Particle filter extracts arrivals } \\
\text { - Ambiguity surface search }\end{array}$ & - Assumes isovelocity profile \\
\hline $5(2009)$ & Fingerprinting & $\begin{array}{l}\text { - Fingerprint database } \\
\text { - Broadband signal }\end{array}$ & $\begin{array}{l}\text { - Database of modeled CIRs } \\
\text { - Pattern matching of CIR measurements at } \\
\text { different frequencies }\end{array}$ & $\begin{array}{l}\text { - Multiple receivers } \\
\text { - Maintenance of fingerprint } \\
\text { database in ocean environments }\end{array}$ \\
\hline $20(1990)$ & $\begin{array}{l}\text { Matched field } \\
\text { processing }\end{array}$ & $\begin{array}{l}\text { - Hydrophone array } \\
\text { - Known environmental parameters }\end{array}$ & $\begin{array}{l}\text { - Acoustic field replica computation } \\
\text { - Gridded virtual source positioning } \\
\text { - ML or Bartlett processing }\end{array}$ & $\begin{array}{l}\text { - Requires multiple receivers to } \\
\text { decrease ambiguity }\end{array}$ \\
\hline $22(2014)$ & $\begin{array}{l}\text { Radio UWB } \\
\text { fingerprinting }\end{array}$ & $\begin{array}{l}\text { - UWB radio modeling to pre- } \\
\text { compute field dictionary } \\
\text { - Multiple antennas }\end{array}$ & $\begin{array}{l}\text { - UWB fingerprinting } \\
\text { - CS solution via orthogonal matching pursuit } \\
\text { and Lasso-II }\end{array}$ & $\begin{array}{l}\text { - Multiple antennas } \\
\text { - Limited to short-range } \\
\text { localization }\end{array}$ \\
\hline $21(2017)$ & $\begin{array}{l}\text { CS, matched field } \\
\text { proc. }\end{array}$ & $\begin{array}{l}\text { - Hydrophone array } \\
\text { - Known environmental parameters }\end{array}$ & $\begin{array}{l}\text { - Acoustic field replica computation } \\
\text { - CS solution via basis pursuit and Lasso }\end{array}$ & - Multiple receivers \\
\hline 23 (2012) & Range estimation & $\begin{array}{l}\text { - Single receiver } \\
\text { - Known waveguide invariant }\end{array}$ & $\begin{array}{l}\text { - Source velocity computation } \\
\text { - Identification of point closest to receiver }\end{array}$ & - Range-only \\
\hline 25 (2018) & Fingerprinting & - Known environmental parameters & $\begin{array}{l}\text { - CIR computation over a fine two-dimensional } \\
\text { vertical grid } \\
\text { - Matching with measurements from multiple } \\
\text { transmitters }\end{array}$ & $\begin{array}{l}\text { - Multiple projectors } \\
\text { - Fixed-bearing localization }\end{array}$ \\
\hline $26(2014)$ & $\begin{array}{l}\text { Acoustics-aided } \\
\text { inertial tracking }\end{array}$ & - External bearing/speed sensor & $\begin{array}{l}\text { - Extract phase from train of sine waves } \\
\text { - Solves inverse problem to determine AUV } \\
\text { location }\end{array}$ & $\begin{array}{l}\text { - Requires accurate bearing/ } \\
\text { speed measurements }\end{array}$ \\
\hline
\end{tabular}

correspond to the same location or to different locations in case the AUV is moving. Finally, we apply an efficient, lowcomplexity tracking mechanism in order to filter all matching locations found, and to obtain a source trajectory estimate.

Figure 3 presents an example of the output of four subsequent location estimates. Figures 3(a)-3(d) show a map of the scenario. Our single receiver is shown as a centrally located square, whereas the AUV that moves along the trajectory is represented as a black line. At each of the positions marked by two concentric circles, the AUV emits a signal that is employed by the receiver to compute location estimates as explained above. In Figs. 3(a)-3(d), these location estimates are represented as gray crosses, where a darker gray shade indicates a higher confidence. The algorithm outputs multiple estimates for each AUV location, each with different levels of confidence (higher confidence is represented using a darker gray shade in Figs. 3(a)-3(d)]. Note that the point of highest confidence may not be the closest to the actual AUV location.
To rule out spurious estimates, we order the computed locations into a trellis [Fig. 3(e)], and run a forward-backward path search procedure similar to the Viterbi algorithm. In this case, the black path in Fig. 3(e) is selected, corresponding to the trajectory shown in Fig. 3(f).

\section{B. Preliminary assumptions and setup}

The first step to localize the AUV is to detect its periodic pinger signals. We assume that no prior information is available about the location, the instantaneous speed, or the trajectory of the AUV, and that the AUV does not collaborate to the localization process. Hence, a solution based on updating the parameters of a dynamic model for the AUV through filtering is not an option in our scenario. We only assume that the emitted signal's waveform is either known, or can be reliably estimated, such that the CIR can be evaluated. By this, we take into account received multipath, but ignore interference. Hence, our method is geared into the

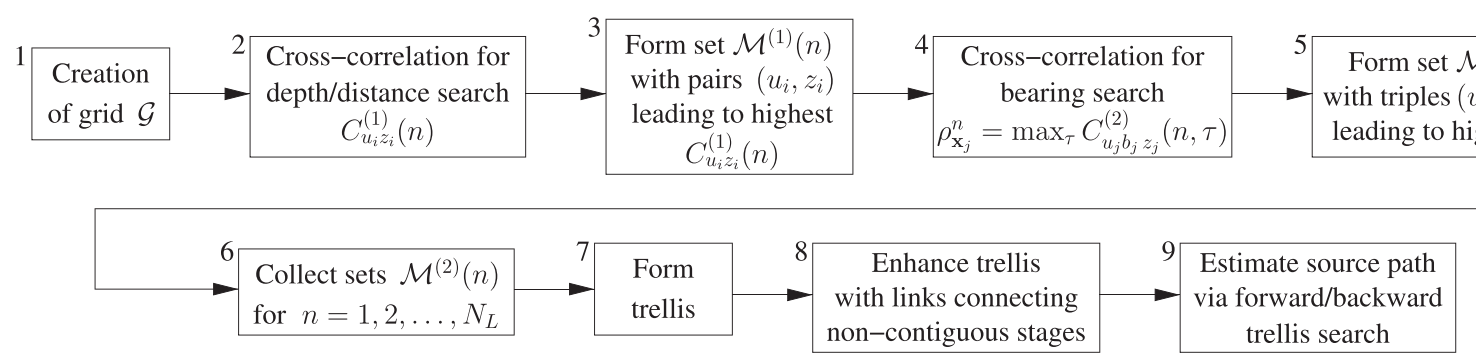

FIG. 1. Block diagram of the AUV location and path estimation algorithm. 


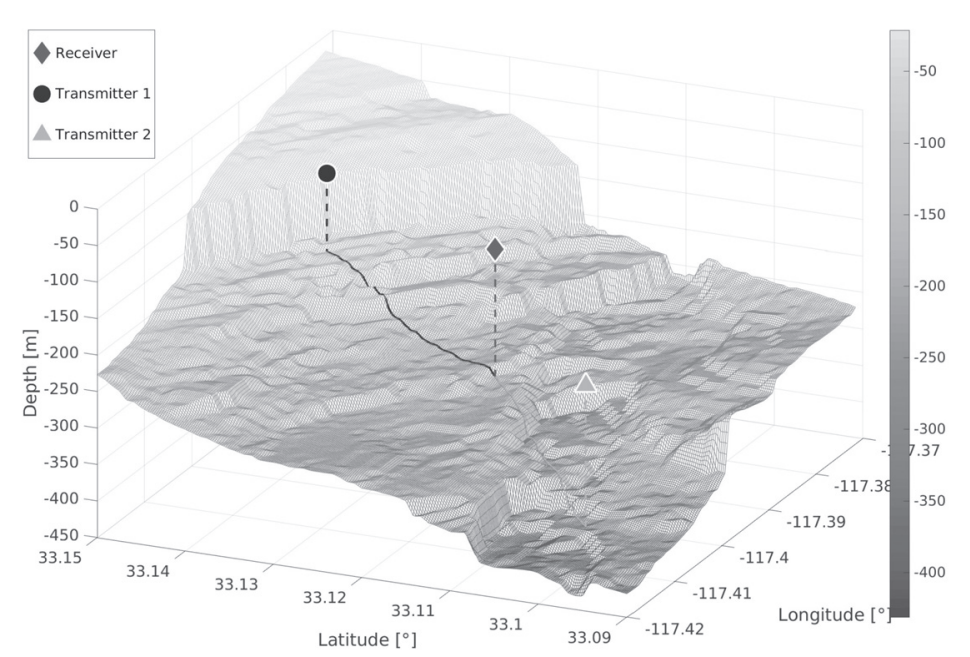

(a) Map of the San Diego bay area, showing a variable bathymetry, the location of the receiver and of two transmitters, and the sea bottom profiles between each transmitter and the receiver.

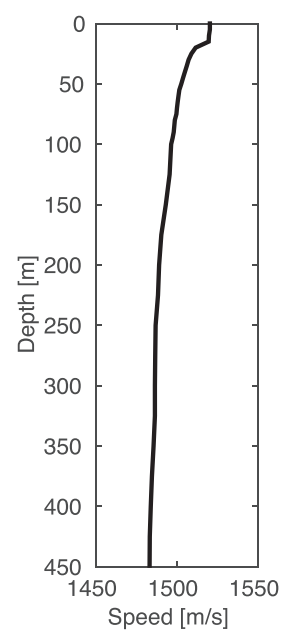

(b) Example of SSP

measured in the area.

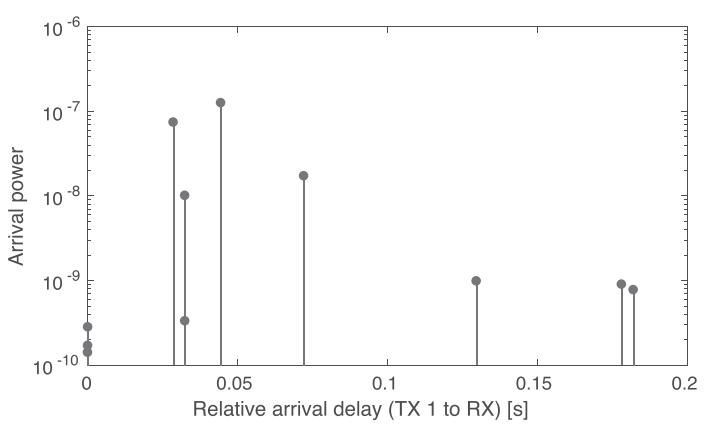

(c) CIR from transmitter 1 to the receiver.

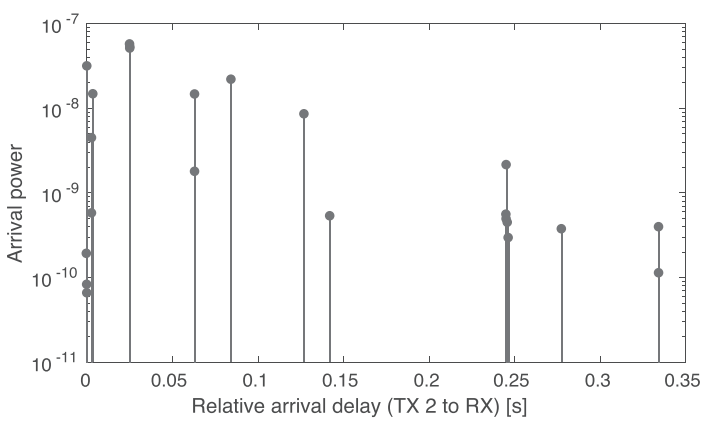

(d) CIR from transmitter 2 to the receiver.

FIG. 2. (Color online) Illustration of our single-receiver localization method. When the environment is sufficiently diverse (a), the CIRs differ significantly across different locations (c) and (d). This can be leveraged for localization.

localization of a single source. We assume that an initial survey has been carried out in order to measure the bathymetry of the area surrounding the moored receiver with a fine resolution. The $1-\mathrm{m}$ resolution obtained by a $400-\mathrm{kHz}$ multibeam sonar (see our experimental results in Sec. V) is more than sufficient in this respect. We further require periodic direct or indirect measurements of the local SSP.

The area explored to localize the AUV is limited by the coverage of the bathymetry measurements, by the reception capabilities of the receiver, and by constraints on the emitter's source level. This yields a bounded depth range between $z_{\min }^{\mathrm{s}}$ and $z_{\max }^{\mathrm{s}}$. We further assume the AUV is moving at an absolute maximum speed of $v_{\max }^{\mathrm{s}}$, known to the receiver. This leads to an expectation on the maximum distance traveled by the AUV between two subsequent emissions. We note that the knowledge of the AUV's maximum speed is not strictly required, but the availability of this information improves the performance and greatly reduces the complexity of our method. At different locations, indexed by $n=1, \ldots, N_{L}$, the source emits acoustic signals that are detected by the receiver along with each significant multipath arrival. The locations are expressed in terms of a cylindrical coordinate system as $\mathbf{x}_{n}^{\mathrm{s}}=\left(u_{n}^{\mathrm{s}}, b_{n}^{\mathrm{s}}, z_{n}^{\mathrm{s}}\right)$ where, at location index $n, u_{n}^{\mathrm{s}} \in\left[0, u_{\max }\right]$ is the great-circle distance in meters between the receiver and the source, $b_{n}^{\mathrm{s}} \in\left[0^{\circ}, 360^{\circ}\right)$ is the bearing of the AUV with respect to the receiver (i.e., the angle at which the receiver sees the source, measured clockwise from due north) and $z_{n}^{\mathrm{s}} \in\left[z_{\min }^{\mathrm{s}}, z_{\max }^{\mathrm{s}}\right]$. We define the AUV's path as the ordered source location sequence $\left\{\mathbf{x}_{n}^{\mathrm{s}}, \ldots, \mathbf{x}_{N_{L}}^{\mathrm{s}}\right\}$.

The database of modeled CIRs set up by the receiver is computed at all points of a cylindrical grid designed to span the ranges $\mathcal{U}=\left\{\delta_{u}, 2 \delta_{u}, \ldots, u_{\max }\right\}$, the bearing angles $\mathcal{B}=\left\{\delta_{b}, 2 \delta_{b}, \ldots 360^{\circ}\right\}$, and the depth values $\mathcal{Z}=\left\{z_{\text {min }}^{\mathrm{s}}, z_{\text {min }}^{\mathrm{s}}\right.$ $\left.+\delta z, \ldots, z_{\max }^{\mathrm{s}}\right\}$. The set of grid points is then defined as $\mathcal{G}=\mathcal{U} \times \mathcal{B} \times \mathcal{Z}$, where we denote $\mathbf{g}_{u_{i} b_{z_{i}}} \in \mathcal{G}$ as the $i$ th grid point, $i=1, \ldots,|\mathcal{G}|$. This corresponds to the first box in Fig. 1 .

\section{AUV location estimation}

For each grid point $\mathbf{g}_{u_{i} b_{i} z_{i}}$, the receiver models the expected CIR using a propagation model. For this purpose, we employ the Bellhop ray tracing software. Bellhop is an established solution to numerically solve pressure wave propagation equations by taking into account boundary conditions. In particular, Bellhop can factor in, among others: the SSP at multiple points throughout the water body section that joins the transmitter to the receiver; the relevant bathymetry in the area, including abrupt changes; the shape of surface waves; and the geo-acoustic properties of the sea bottom sediments. Bellhop has been used to model acoustic 


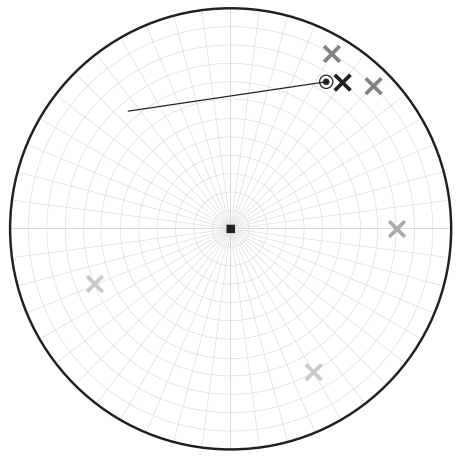

(a) Step 1

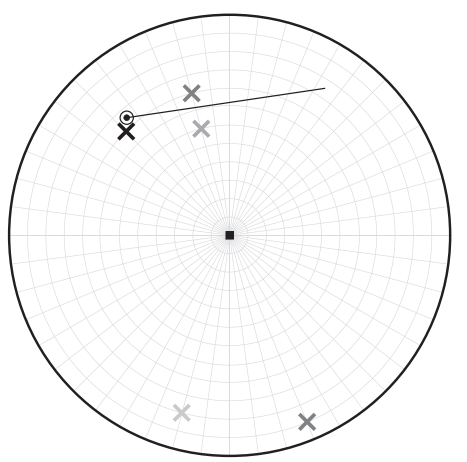

(d) Step 4

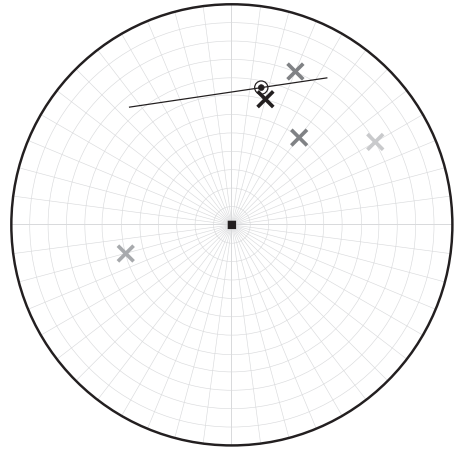

(b) Step 2

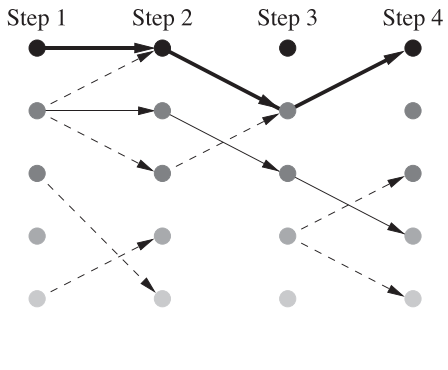

(e) Trellis

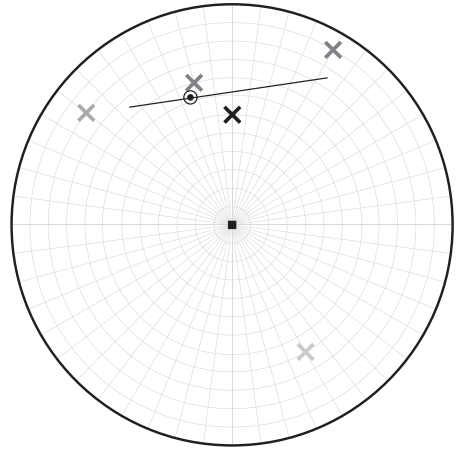

(c) Step 3

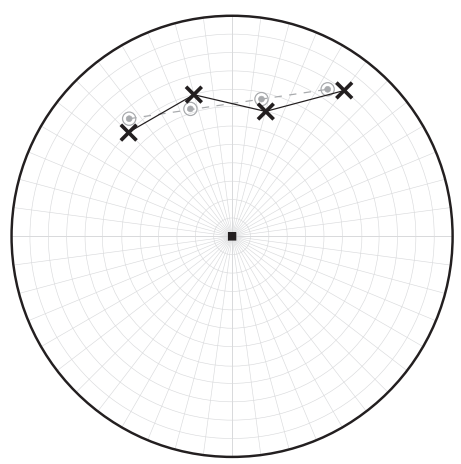

(f) Trajectory estimate

FIG. 3. High-level illustration of the key idea behind our single-receiver localization process. (a)-(d) show a sound source moving along a straight trajectory. At four locations, the source emits a signal. The receiver (located at the center of the area) measures the CIR and compares it against a database of modeled channel responses. This translates into the location estimates indicated by the crosses, where a darker gray shade indicates higher confidence. A trellis search algorithm (e) is then applied to find the most likely source path (f).

channels in different communication contexts and served as the basis for more complex models (e.g., see Refs. 39 and 40). In our context, Bellhop yields accurate time-of-arrival information for each acoustic path and sufficiently accurate complex amplitude information, so that the outcome of correlation operations can be trusted. We will show that Bellhop offers sufficiently reliable CIR modeling in a sea trial in Sec. V.

The output of Bellhop includes a list of expected multipath arrivals, along with their amplitude, phase, delay, and reception angle. Moreover, for each arrival, Bellhop reports the list of bottom and surface reflections it incurred. This information is employed to construct two modeled responses, namely a partial CIR $h_{u_{i} z_{i}}^{(1)}(t)$, containing only the specular and surface-reflected arrivals, ${ }^{41}$ and the complete CIR $h_{u_{i} b_{i} z_{i}}^{(2)}(t)$. As the specular and surface-reflected arrivals are practically independent of the bearing of the AUV relative to the receiver and rather depend only on the SSP, on $u_{i}$ and on $z_{i}$, the subscript $b_{i}$ has been dropped in $h_{u_{i} z_{i}}^{(1)}(t)$.

From the modeled CIRs, the receiver obtains two separate fingerprints, $h_{u_{i} z_{i}}^{(1)}$ and $h_{u_{i} b_{i} z_{i}}^{(2)}$. When the source is at location $\mathbf{x}_{n}$, its emitted signal is received as

$$
r_{n}(t)=\widehat{h}_{u_{n} b_{n} z_{n}}(t) \otimes s(t)+\nu(t),
$$

where $\widehat{h}_{u_{n} b_{n} z_{n}}(t)$ is the CIR estimated from a received signal, $s(t)$ is the emitted signal waveform, $\nu(t)$ is the ambient noise, and $\otimes$ denotes convolution. The receiver then computes

$$
\begin{aligned}
& f_{u_{i} z_{i}}^{(1)}=h_{u_{i} z_{i}}^{(1)}(t) \otimes s(t), \\
& f_{u_{j} b_{j} z_{j}}^{(2)}=h_{u_{j} b_{j} z_{j}}^{(2)}(t) \otimes s(t),
\end{aligned}
$$

and matches $r_{n}(t)$ against the fingerprints $f_{u_{i} z_{i}}^{(1)}$ and $f_{u_{j} b_{j} z_{j}}^{(2)}$ corresponding to the grid points in $\mathcal{G}$ as follows.

For each point $\left(u_{i} z_{i}\right)$ in the grid, we compute the normalized correlation

$$
C_{u_{i} z_{i}}^{(1)}(n)=\frac{\int_{0}^{+\infty} r_{n}(t) f_{u_{i} z_{i}}^{(1)}(t-\tau) \mathrm{d} t}{\left(\int_{0}^{T} r_{n}(t)^{2} \mathrm{~d} t \int_{0}^{+\infty} f_{u_{i} z_{i}}^{(1)}(t)^{2} \mathrm{~d} t\right)^{1 / 2}},
$$

where $T$ is the signal's duration and $\tau$ is the time epoch corresponding to the correlation maximum. Note that in Eq. (3) we perform a normalized correlation to reduce the sensitivity to CIRs characterized by different power attenuation. Define $\mathcal{M}^{(1)}(n)$ as the set of all pairs $\left(u_{j}, z_{j}\right)$ corresponding to those $C_{u_{i} z_{i}}^{(1)}(n)$ that exceed a certain threshold $\Theta_{D}, \forall\left(u_{i}, z_{i}\right) \in \mathcal{G}$, where we set $\Theta_{D}$, using the analysis in Ref. 42. We remark that we do not limit set $\mathcal{M}^{(1)}(n)$ to contain just the coordinates of the single grid point yielding the maximum correlation. In fact, the estimation of the correct distance and depth may still be hindered by the lack of, e.g., the specular arrival, which can occur in the presence of SSP patterns with a sufficiently steep gradient and for a sufficiently large distance 
between the AUV and the receiver (e.g., see the example on p. 46 of Bellhop's manual ${ }^{38}$ ). Including a number of possible matching locations yields more robustness against such errors. The above steps correspond to boxes 2 and 3 in Fig. 1.

For each $\left(u_{j}, z_{j}\right) \in \mathcal{M}^{(1)}(n)$ and $\forall b \in \mathcal{B}$, we compute the following normalized correlations:

$$
C_{u_{j} b_{j} z_{j}}^{(2)}(n, \tau)=\frac{\int_{0}^{+\infty} r_{n}(t) f_{u_{j} b_{j} z_{j}}^{(2)}(t-\tau) \mathrm{d} t}{\left(\int_{0}^{T} r_{n}(t)^{2} \mathrm{~d} t \int_{0}^{+\infty} f_{u_{j} b_{j} z_{j}}^{(2)}(t)^{2} \mathrm{~d} t\right)^{1 / 2}} .
$$

Call

$$
\rho_{\mathbf{x}_{j}}^{n}=\max _{\tau} C_{u_{j} b_{j z_{j}}}^{(2)}(n, \tau),
$$

and define $\mathcal{M}^{(2)}(n)$ as the set of all triples $\mathbf{p}_{k}=\left(u_{k}, b_{k}, z_{k}\right)$ corresponding to the $R^{(2)}$ highest values of $\rho_{\mathbf{p}_{k}}^{n} \forall\left(u_{j}, z_{j}\right)$ $\in \mathcal{M}^{(2)}(n)$ and $\forall b \in \mathcal{B}$, where $R^{(2)}$ is a user-defined parameter (in our performance evaluation, we set $R^{(2)}=70$ ). The above steps correspond to boxes 4 and 5 in Fig. 1.

In ideal conditions, e.g., with an extremely dense grid $\mathcal{G}$, in the absence of noise, and with perfect environmental information, it would be enough to limit set $\mathcal{M}^{(2)}(n)$ to the coordinates of the point $\mathbf{p}_{k}=\left(u_{k}, b_{k}, z_{k}\right)$ for which $\rho_{\mathbf{p}_{k}}^{n}$ is highest. However, in any practical scenario, the grid point closest to the actual position of the AUV might not yield the highest correlation due to noise, outdated environmental information, or a combination of both. In this perspective, it is convenient to set $R^{(2)}$ to some large value. On the other hand, it is computationally infeasible to have an exceedingly large set $\mathcal{G}$. For this reason, we reduce the complexity of the search whenever possible by limiting the location search area through a bound on the distance between the AUV and the receiver. For example, if the source level is known, this bound can be obtained based on a received signal strength indicator (RSSI). ${ }^{43}$ Furthermore, in the following we present a filtering scheme that reduces the complexity of path estimation.

\section{AUV path estimation}

After determining the possible matching locations $\mathcal{M}^{(2)}(n)$ for $n=1, \ldots, N_{L}$, we proceed to find the most likely sequence of AUV's locations among all possible options using a path estimation algorithm. Without prior information about the AUV motion pattern, we avoid assuming a dynamic model solved by filtering, but rather work on a trellis such as the one shown in Fig. 4. The trellis has $N_{L}$ stages, one for each transmission received from the AUV. In each stage, different nodes represent different estimated locations, so that the first stage of the trellis represents all location estimates for the first detected signal from the AUV [set $\mathcal{M}^{(2)}(1)$ ], the second stage contains the estimates in set $\mathcal{M}^{(2)}(2)$, and so forth until the last stage, which contains the estimates in $\mathcal{M}^{(2)}\left(N_{L}\right)$. We assign a confidence index to each node in the trellis [the value of the normalized

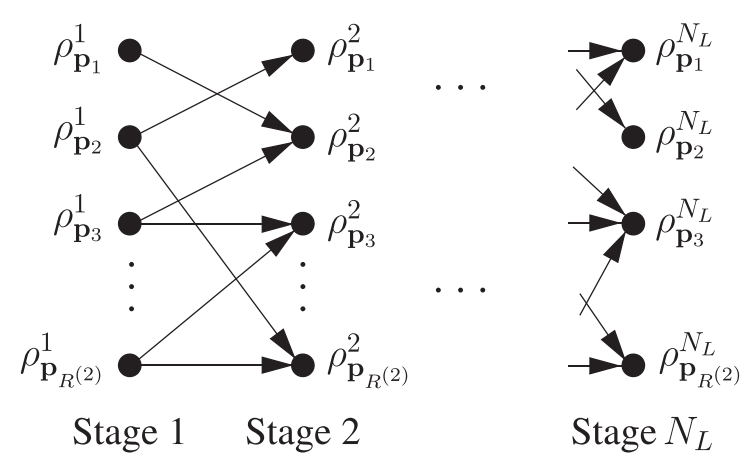

FIG. 4. Example of trellis employed by the tracking algorithm for the source path estimation. Each node represents a location estimate. Trellis links exist only among locations that are closer than the maximum distance $d_{\max }$ covered by the AUV when traveling at full speed between subsequent signal transmissions.

cross-correlation between the modeled and measured channels, see Eq. (5)], and organize them into a $R^{(2)} \times N_{L}$ matrix T (boxes 6 and 7 in Fig. 1). Both the nodes in the $i$ th trellis stage and the entries in the $i$ th column of $\mathbf{T}$ are sorted in order of decreasing confidence, i.e., $[\mathbf{T}]_{1, i}=\rho_{\mathbf{p}_{1}}^{i},[\mathbf{T}]_{2, i}=\rho_{\mathbf{p}_{2}}^{i}$, $[\mathbf{T}]_{R^{(2), i}}=\rho_{\mathbf{p}_{R^{(2)}}}^{i}$, and

$$
\rho_{\mathbf{p}_{1}}^{i}>\rho_{\mathbf{p}_{2}}^{i}>\cdots>\rho_{\mathbf{p}_{R}(2)}^{i} .
$$

\section{Setting the path weights}

The objective of path estimation is to find the best sequence of nodes across consecutive trellis stages. To that end, a link exists between an entry in stage $n$ and an entry in stage $n+1$ if the locations represented by these nodes are closer than the maximum distance the AUV could cover when traveling at full speed $v_{\max }^{\mathrm{s}}$ between the $n$th and the $(n+1)$ th signal detections (recall that the maximum absolute speed is assumed to be known). Formally, call $e_{\ell_{n} \ell_{n+1}}$ the edge that connects the $\ell_{n}$ th node at stage $n$ in the trellis (entry in column $n$ of $\mathbf{T}$ ) and the $\ell_{n+1}$ th entry at column $n+1$. Call $A\left(e_{\ell_{n} \ell_{n+1}}\right)=\mathbf{p}_{\ell_{n}}$ and $S\left(e_{\ell_{n} \ell_{n+1}}\right)=\mathbf{p}_{\ell_{n+1}}$ the ancestor and the successor of edge $e_{\ell_{n} \ell_{n+1}}$, respectively. Define the edge weight as

$\sigma\left(e_{\ell_{n} \ell_{n+1}}\right)= \begin{cases}1, & \text { if } d\left(\mathbf{p}_{\ell_{n}}, \mathbf{p}_{\ell_{n+1}}\right) \leq d_{\max } \\ \frac{d_{\max }}{d\left(\mathbf{p}_{\ell_{n}}, \mathbf{p}_{\ell_{n+1}}\right)}, & \text { if } d_{\max }<d\left(\mathbf{p}_{\ell_{n}}, \mathbf{p}_{\ell_{n+1}}\right) \leq 1.5 d_{\max } \\ 0, & \text { otherwise, }\end{cases}$

where $d(\mathbf{x}, \mathbf{y})=\|\mathbf{x}-\mathbf{y}\|_{2}$ is the Euclidean distance between locations $\mathbf{x}$ and $\mathbf{y}, t_{n}$ and $t_{n+1}$ are the reception epochs of the $n$th and $(n+1)$ th detected signals, respectively, and $d_{\max }$ $=v_{\max }^{\mathrm{s}}\left(t_{n+1}-t_{n}\right)$ is the maximum distance that the AUV could have traveled between time epochs $t_{n}$ and $t_{n+1}$. Only edges with non-zero weights are considered for path estimation. To form a continuous path, we require connected edges. In particular, if for edge $e_{\ell_{n} \ell_{n+1}}$ it occurs that its ancestor $\mathbf{p}_{\ell_{n}}$ is not a successor of any edge $e_{\ell_{n-1} \ell_{n}}$, or that its successor $\mathbf{p}_{\ell_{n+1}}$ is 
not an ancestor of any edge $e_{\ell_{n+1}, \ell_{n+2}}$, then the weight of edge $e_{\ell_{n} \ell_{n+1}}$ is set as zero, and the edge is removed from the trellis.

We remark the similarities between the path estimation algorithm and the Viterbi algorithm for tracking within a trellis (see also Ref. 44). While the Viterbi algorithm would yield the optimal solution, it would include all grid points in $\mathcal{G}$ in each stage of the trellis. This would require $|\mathcal{G}|$ entries in each column of $\mathbf{T}$, which would compound to a huge state space and imply an exceedingly high computational complexity, especially if $|\mathcal{G}|$ is very large. In addition, solving through the Viterbi algorithm would require an estimation for the emission and transition probabilities, which involves some hard assumptions on the CIR and noise models. Instead, our version relies on confidence indices and makes it possible to trim the state space according to physical movement constraints. This leads to a significant performance improvement and to a feasible path estimation complexity.

\section{Finding the best path}

Let $\mathcal{E}(n)=\left\{e_{\ell_{n} \ell_{n+1}}\right\}$ be the set of edges that link a node in stage $n$ of the trellis to a node in stage $n+1$, and use Eq.

(7) to define the following metric for each edge:

$$
\lambda\left(e_{\ell_{n} \ell_{n+1}}\right)=\rho_{\mathbf{p}_{\ell_{n}}}^{n} \rho_{\mathbf{p}_{\ell_{n+1}}}^{n+1} \sigma\left(e_{\ell_{n} \ell_{n+1}}\right),
$$

where the confidence indices are taken from T. Define a generic path on the trellis as

$$
\Psi=\left\{e_{1}, \ldots, e_{N_{L}}\right\},
$$

where $e_{i}$ is shorthand for $e_{\ell_{i} \ell_{i+1}} \in \mathcal{E}(i)$, and all edges are such that $S\left(e_{i}\right)=A\left(e_{i+1}\right), i=1, \ldots, N_{L}-1$. Define the overall path metric as

$$
\Lambda(\Psi)=\frac{\prod_{i=1}^{N_{L}-1} \lambda\left(e_{i}\right)}{\prod_{i=1}^{N_{L}-2} \rho_{S\left(e_{i}\right)}^{i}}
$$

i.e., as the product of the confidence metrics for all edges that belong to the path, divided by the confidence of intermediate nodes in order to avoid accounting for them twice. The path estimate is finally found as

$$
\widehat{\Psi}=\underset{\Psi}{\arg \max } \Lambda(\Psi)
$$

and we indicate the sequence of locations traversed by $\widehat{\Psi}$ as $\left\{\widehat{\mathbf{x}}_{1}, \widehat{\mathbf{x}}_{2}, \ldots, \widehat{\mathbf{x}}_{N_{L}}\right\}$.

As a means of measuring the discrepancy between the true and the estimated sequence of AUV's locations, we consider the root-mean-square (RMS) point-wise distance between corresponding points of the true and estimated paths. Formally,

$$
\varepsilon_{\hat{\Psi}}^{d}=\left(\frac{1}{N_{L}} \sum_{n=1}^{N_{L}} d\left(\widehat{\mathbf{x}}_{n}, \mathbf{x}_{n}^{\mathrm{s}}\right)^{2}\right)^{1 / 2} .
$$

We also convey the source bearing estimation effectiveness of our approach via the bearing error

$$
\varepsilon_{\hat{\Psi}}^{a}=\frac{1}{N_{L}} \sum_{n=1}^{N_{L}}\left|\widehat{b}_{n}-b_{n}^{\mathrm{s}}\right|,
$$

where $d(\cdot, \cdot)$ denotes the distance between two points in the cylindric coordinate system.

\section{Refinement}

In this section, we present two refinements to the above algorithm. The first refinement relates to the possible case that there exists no edge with a non-zero weight connecting two trellis stages $n$ and $n+1$. This would lead to a partitioning of the trellis. We correct for these cases by allowing stage $n-1$ to directly connect to stage $n+1$. Specifically, the corresponding edge $e_{\ell_{n-1} \ell_{n+1}}$ will have a weight equal to

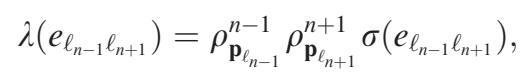

where $\sigma(\cdot)$ is the same as in Eq. (7).

The above recovery mechanism is further enhanced to handle cases of broader trellis partitioning due to bursts of errors. These bursts are caused by strong noise from, e.g., a nearby vessel or waves, or due to erroneous bathymetry information at some locations. The results of such bursts are sets of short paths for which the maximization in Eq. (11) is not optimal, i.e., the problem becomes non-convex. Considering this case, we increase the number of paths in $\Psi$ through our second refinement procedure as follows.

We start by observing that from the perspective of path finding we can calculate paths by taking sets of estimated locations either in the order they occur in time, or by reversing this order. In other words, the trellis stages in Fig. 4 and the corresponding columns in $\mathbf{T}$ can be flipped, such that the first contains location estimates in $\mathcal{M}^{(2)}\left(N_{L}\right)$, the second contains the estimates in $\mathcal{M}^{(2)}\left(N_{L}-1\right)$, and so forth until the last column, which contains the samples in $\mathcal{M}^{(2)}(1)$. Call $\Psi_{F}$ a forward path on the trellis traversing locations $\left\{\mathbf{x}_{1}, \mathbf{x}_{2}, \ldots, \mathbf{x}_{N_{L}}\right\}$, and call $\Psi_{B}$ a backward path computed on the reversed trellis, traversing locations $\left\{\mathbf{y}_{N_{L}}, \mathbf{y}_{N_{L}-1}, \ldots, \mathbf{y}_{N_{1}}\right\}$. If $\widehat{\Psi}_{F}$ and $\widehat{\Psi}_{B}$ are the best forward and backward paths according to Eq. (11), respectively, we set the final path estimate $\widehat{\Psi}=\widehat{\Psi}_{F}$ if $\Lambda\left(\widehat{\Psi}_{F}\right)>\Lambda\left(\widehat{\Psi}_{B}\right)$, and $\widehat{\Psi}=\widehat{\Psi}_{B}$ otherwise. In case of significant interruptions in the trellis structure, the above scheme increases the probability to find the correct path. The scheme is also beneficial if the estimate of the initial location on the forward path is incorrect, making the path search diverge to a mostly wrong sequence of locations. In case of a well-connected trellis, instead the scheme is likely to find the same path twice, with no effect on the accuracy of the algorithm.

The complexity of the algorithm relates to the number of correlation operations and to the trellis search. For each received source signal, the algorithm computes $\kappa|\mathcal{G}|+\mathcal{O}\left(\left|\mathcal{M}^{(1)}(n)\right|\right)$ $\approx \kappa|\mathcal{G}|$ correlations in order to extract the possible position estimates in set $\mathcal{M}^{(2)}(n)$, where $\kappa$ is a proportionality factor that accounts for the search space reduction enabled, e.g., by RSSI 
bounding considerations as mentioned in Sec. III C. For a signal of bandwidth-time duration product $B T$, the complexity of each normalized cross correlation is $\mathcal{O}\left(B^{2}\right)$. With $\mathcal{O}\left(N_{L}\left|\mathcal{M}^{(2)}(n)\right|\right)$ operations for the trellis search, the overall complexity is $\mathcal{O}\left(N_{L}\left|\mathcal{M}^{(2)}(n)\right|+\kappa|\mathcal{G}| B^{2}\right)$. Comparing this with the complexity of the Viterbi algorithm, i.e., $\mathcal{O}\left(N_{L}|\mathcal{G}|^{2}\right)$ (see also Ref. 32), a significant complexity reduction exists.

\section{E. Discussion}

Our method considers the practical case of observing an unknown target. This target can move in any dynamic pattern and even irregularly. Hence, we avoid evaluating its position through filtering, and rather follow a trellis search approach over the confidence indices. This also means that the path found from all feasible solutions is the one with maximum overall confidence index, and thus isolated positions associated to a high confidence value will not be chosen. This is appropriate since we are looking for a systematic solution, rather than an individual match. Our solution for the trellis search takes a suboptimal approach by taking into account sets of only two nodes. This has the drawback that a single node in the trellis may have a higher impact than it should. Yet, without prior knowledge of the target and to keep the calculations feasible we avoid other solutions in the form of, e.g., dynamic programming. Further, we note that the accuracy of our method depends on the quality of the channel estimation process, which improves with the bandwidth of the emitted signal.

For channel modeling, we use the bathymetry and the SSP. Without up-to-date information about instantaneous sea conditions, we avoid a time-varying propagation model and use instead a static model. Instead, the time-variation of the channel is taken into account by the AUV's motion, both by calculating different channels for different locations, and by using the maximum velocity $v_{\max }^{\mathrm{s}}$. This parameter trades off complexity with performance, as higher values for the maximum speed correspond to additional possible paths in the considered trellis. Another significant assumption is the ability to estimate the channel from the received signals. Clearly the performance of our approach depends on the accuracy of such estimation. While channel estimation is beyond the scope of this work, possible techniques for such an estimation can be rake receivers, ${ }^{45}$ blind source separation, ${ }^{46}$ or cyclo-stationary analysis, ${ }^{47}$ to name a few options.

\section{SIMULATION RESULTS}

\section{A. Scenario and parameters}

For our simulations, we consider a portion of the San Diego bay area, off the coast of southern California, which is a well-explored area. We place the receiver at the coordinates $\left[32.9390^{\circ} \mathrm{N}, 117.2816^{\circ} \mathrm{W}\right]$. We take the area's bathymetry data from the U.S. Coastal Relief model ${ }^{48}$ (revealing that the average depth in the area is about $50 \mathrm{~m}$ ), and employ an SSP sample taken at the observed area. The SSP has a downward-refractive shape, typical of shallow Californian waters during warm seasons, as depicted in Fig. 2(b). We assume that the water surface is flat.
In our simulations, we deploy both the receiver and the source at depths of $10 \mathrm{~m}$. Still, we remark that the receiver is not aware of the source's depth. The simulation starts by deploying the emitting source at random in the area at a range of $500 \mathrm{~m}$ from the receiver. The source then chooses a bearing uniformly at random and moves along the corresponding direction with constant speed chosen at random for the time required to carry out ten transmissions. The locations $\mathbf{x}_{n}^{\mathrm{s}}$ and $\mathbf{x}_{n+1}^{\mathrm{s}}$, where two subsequent emissions take place, are chosen uniformly at random such that $d\left(\mathbf{x}_{n}^{\mathrm{s}}, \mathbf{x}_{n+1}^{\mathrm{s}}\right) \leq d_{\max }$, and we set $d_{\text {max }}=50 \mathrm{~m}$.

The fingerprint grid pre-computed by the receiver spans a total range $u_{\max }=1.5 \mathrm{~km}$ around the receiver, with a resolution of $1 \mathrm{~m}$. The whole azimuthal plane is considered, with a resolution of $1^{\circ}$, and the CIRs are computed for all depths between 5 and $15 \mathrm{~m}$, also with a resolution of $1 \mathrm{~m}$. This choice leads to a total of about $6 \times 10^{6}$ points in set $\mathcal{G}$, and emphasizes the need for our path finding algorithm, as it has much lower complexity than the regular Viterbi algorithm.

The signal transmitted by the source, $s(t)$, is chosen to be a linear chirp signal of duration $100 \mathrm{~ms}$ and bandwidth of $10 \mathrm{kHz}$, centered at a carrier frequency of $12 \mathrm{kHz}$. Based on these signal parameters and using the analysis in Ref. 42, for the computation of Eq. (3) and the formation of set $\mathcal{M}^{(1)}(n)$, we choose $\Theta_{D}=0.1 \forall n$. For each emission from a given source-receiver location pairs, the CIR is computed through Bellhop, ${ }^{38}$ using as parameters the SSP and the available bathymetry samples along the direction from the source to the receiver. The ambient noise at the receiver is modeled as an additive white Gaussian process, whose power is tuned so as to achieve a prescribed signal-to-noise ratio (SNR).

\section{B. Examples}

A sample result from Eq. (3) is shown in Fig. 5(a). We observe a clear peak suggesting that the source is located at a distance of approximately $450 \mathrm{~m}$ from the receiver, at a depth of $10 \mathrm{~m}$. This is due to the presence of all expected specular and surface-reflected arrivals in the received signal. If, e.g., the specular arrival were missing, the correlation peak at $450 \mathrm{~m}$ would not be as high. This is why we consider all three significant peaks, including those at about 300 and $600 \mathrm{~m}$, and for all depths where such peaks exceed $\Theta_{D}$.

To populate set $\mathcal{M}^{(2)}(n)$, we set $R^{(2)}=70$. A sample computation of Eq. (4) for some range-depth pairs in $\mathcal{M}^{(1)}(n)$ is shown in Fig. 5(b). While in this particular case a peak stands out corresponding to the correct bearing of about $150^{\circ}$, often such a favorable result does not occur. The chosen value of $R^{(2)}$ makes it possible to considerably increase the probability that the actual bearing is included in $\mathcal{M}^{(2)}(n)$, while keeping the computational effort controlled.

\section{Localization accuracy for varying SNR}

We start our performance evaluation by running our algorithm in the presence of exact environmental data under different SNR values. The complementary cumulative distribution functions (CCDFs) of the root-mean-square error (RMSE) affecting the distance and bearing estimates are shown in Figs. 6(a) and 6(b), respectively. Thanks to the 


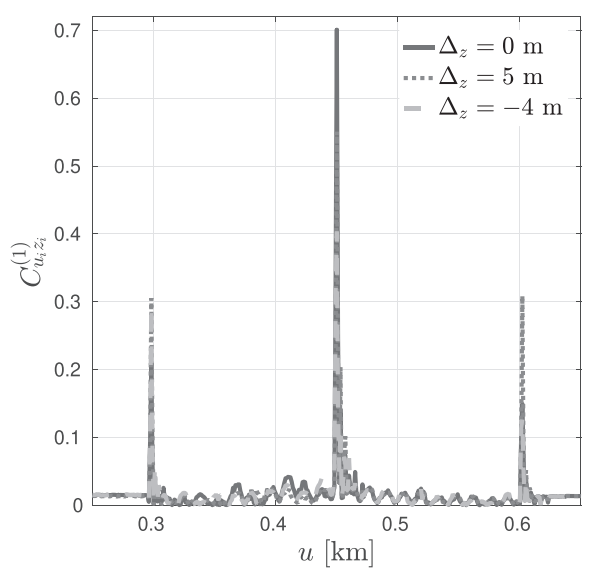

(a) Distance-depth estimation
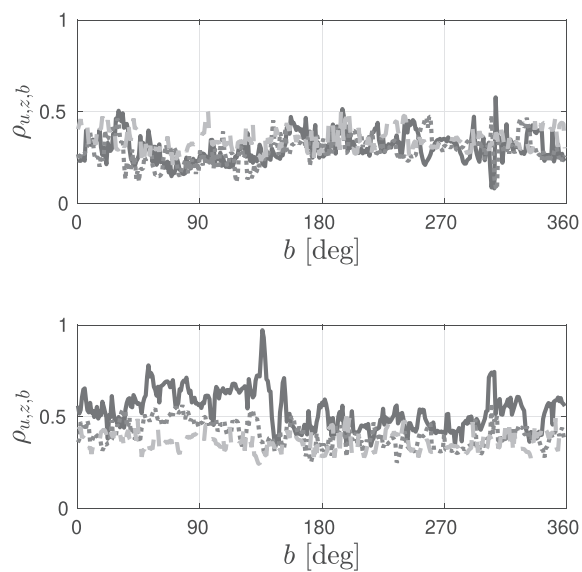

(b) Bearing estimation for a wrong (top) and correct distance (bottom). Key: see Fig. 4a.
FIG. 5. (Color online) Example of correlation values for $\mathbf{x}^{\mathrm{s}}=\left[446 \mathrm{~m}, 150^{\circ}\right.$, $10 \mathrm{~m}]$ at a SNR of $30 \mathrm{~dB}$, for different values of the offset $\Delta z$ between the actual depth and the tested depth. perfect knowledge of both the bathymetry and the SSP in the observed area, neither result shows a significant dependence on the SNR, even after decreasing it to as low as $3 \mathrm{~dB}$, which tends to yield non-negligible spurious peaks in the correlation outputs. We observe that the average RMSE varies from about $120 \mathrm{~m}$ for a SNR of $30 \mathrm{~dB}$, up to about $170 \mathrm{~m}$ for a SNR of $3 \mathrm{~dB}$, with a median error around $80 \mathrm{~m}$, which is satisfactory given the grid resolution employed and the use of a single receiving element. The bearing estimation results show an even higher accuracy, with a mean estimation error $\varepsilon_{a}<20^{\circ}$ even for a SNR of $3 \mathrm{~dB}$, and a median error of less than $10^{\circ}$.

\section{Localization accuracy for imperfect bathymetry data}

The above simulation results show accurate localization for different SNR levels. However, the results are obtained assuming perfect bathymetry and SSP knowledge. In our setting, the receiver is an anchored station, e.g., a marine observatory, and thus we argue that accurate sound speed measurements are possible and do often exist in such marine observatories (e.g., see Ref. 49). Still, while fine-gridded bathymetry mapping can be made around the observatory, small errors and outdated measurements in the resulting depth map may exist. We now explore the sensitivity of our localization method to imperfect bathymetry information.

In the following analysis, to each true bathymetry sample we add an offset drawn uniformly at random in the interval $[-y, y]$, where $y$ (in meters) is a tunable parameter. We collect a Monte Carlo set of 100 source paths and compute the CCDFs of the RMSE for both the distance and the bearing. The results are shown in Figs. 7(a) and 7(b), respectively. We observe that, as expected, mismatched bathymetry data worsens the path estimation performance. However, for a limited offset on bathymetry samples, up to $y=1 \mathrm{~m}$, the median RMS distance

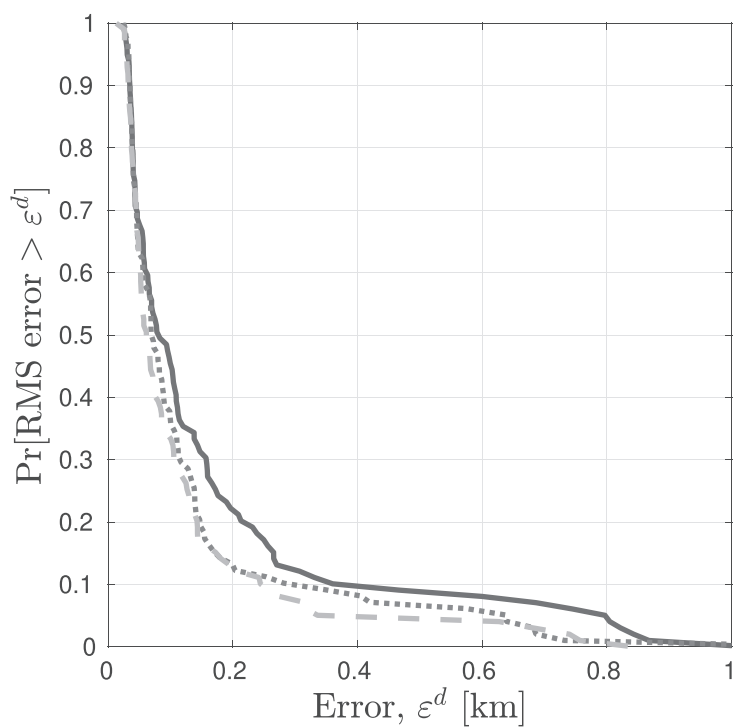

(a) CCDF of the distance RMSE

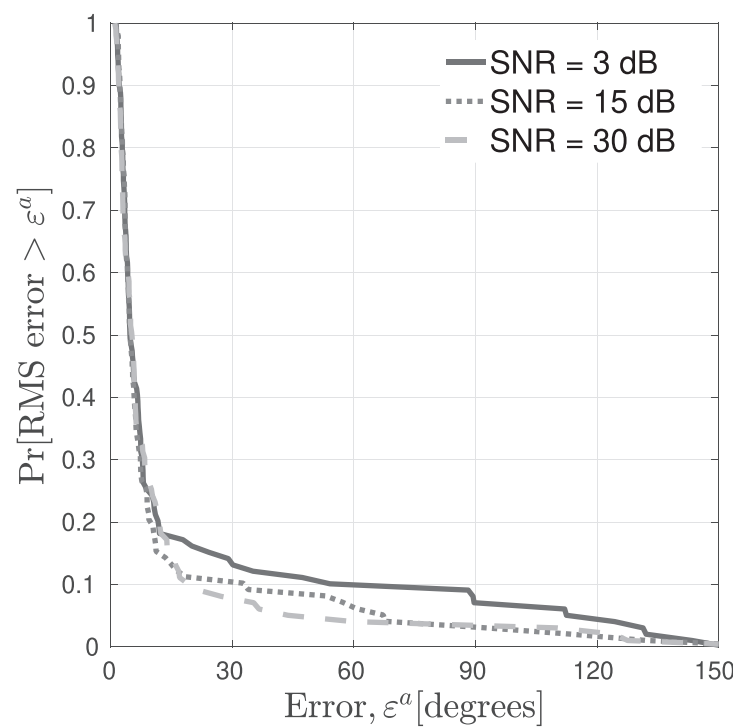

(b) CCDF of the bearing RMSE

FIG. 6. (Color online) Accuracy of the path estimation algorithm in the presence of exact environmental data, for different values of the SNR. Even for low values of the SNR the arrival structure in the CIRs does not change considerably, and has no significant effects on performance. 


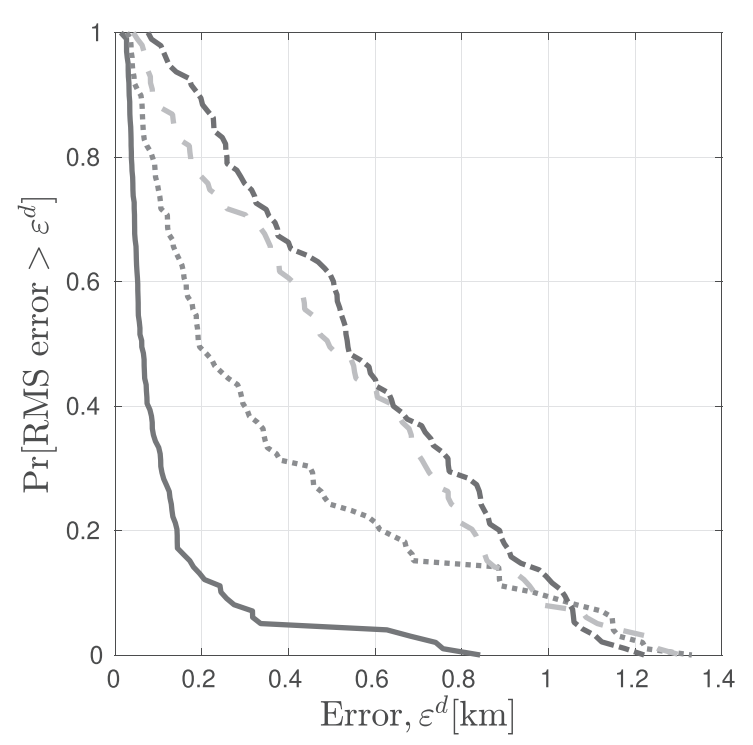

(a) CCDF of the distance RMSE

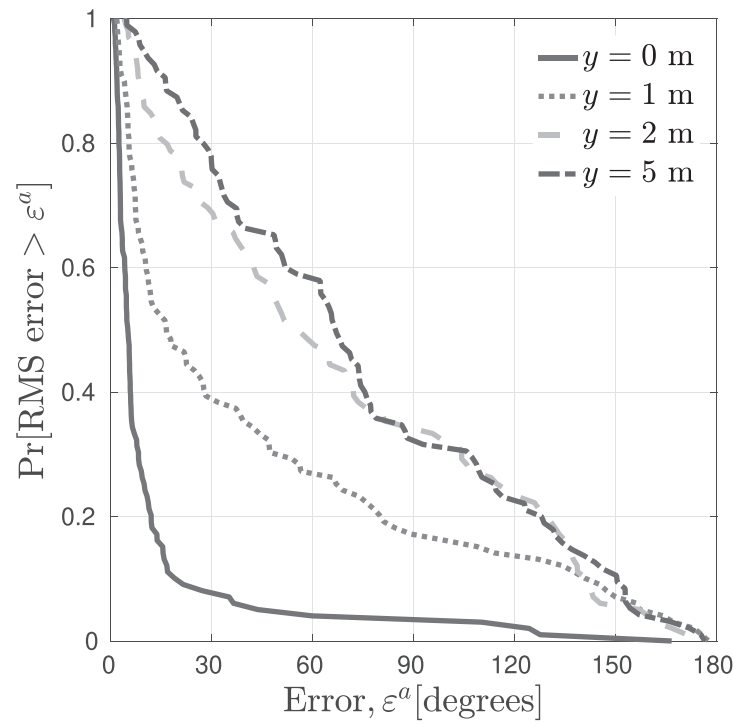

(b) CCDF of the bearing RMSE

FIG. 7. (Color online) Accuracy of the path estimation algorithm in the presence of imperfect bathymetry data. Erroneous bathymetry significantly affects the algorithm's performance. For limited errors $(y=1 \mathrm{~m})$ the results are still viable for several applications.

error remains below $200 \mathrm{~m}$ (or $6 \%$ of the total observed area), which is still a reasonably good result given the presence of a single receiving element. Instead, an error of up to $y=5 \mathrm{~m}$ yields a comparatively worse performance. However, we remark that this is an extreme case, as such an error amounts to about $10 \%$ of the average sea bottom depth in the area, and current sea bottom mapping systems typically ensure submeter bathymetry measurements for depths of less than $200 \mathrm{~m}$ [e.g., this is the case for Kongsberg Maritime's $400 \mathrm{kHz}$ EM 2040 multibeam sonar system we use in our sea experiment (Kongsberg, Kongsberg, Norway)].

Similar conclusions as for the distance-based sensitivity of the algorithm can be drawn also for the bearing estimation error. Figure 7(b) shows that for $y=1 \mathrm{~m}$, the increase in the median bearing estimation error is roughly $20^{\circ}$, and increases to roughly $55^{\circ}$ for $y=5 \mathrm{~m}$. This result emphasizes the need for accurate bathymetry information. Still, we argue that even such rough localization estimates can be instrumental for some applications. For example, security or environmental monitoring systems, where even a rough estimate can trigger a more accurate investigation by human personnel or more complex detection mechanisms; or fauna and habitat monitoring applications, where it is often sufficient to find the approximate path of a vocalizing animal.

\section{E. Localization accuracy for imperfect SSP data}

In order to evaluate the impact of imperfect SSP data on the performance of our algorithm, we add an offset drawn uniformly at random in the interval $[-c, c]$ to each true SSP sample, and carry out Monte Carlo simulations for different values of $c$.

The CCDFs of the distance and bearing RMSE are provided in Figs. 8(a) and 8(b), respectively. While the chosen values for $c$ preserve the general downward-refractive properties of the SSP, even a small value tends to cause significant changes in the structure of multipath arrivals. For $c=0.25 \mathrm{~m} / \mathrm{s}$, we already observe a median distance error of about $250 \mathrm{~m}$ and a median bearing error of about $30^{\circ}$. It could be argued that these values are still practical for rough localization applications, where the only need is to know whether the AUV is practically following a desired trajectory or is falling significantly off track. As expected, increasing $c$ tends to reduce both the distance and the bearing estimation accuracy. This emphasizes the need to maintain SSP estimates updated at the receiver, and to recompute the CIRs in the grid $\mathcal{G}$ in the presence of significant changes.

We finally remark that, besides bathymetry and SSP, high sea states may induce significant surface waves that would also contribute to modifying surface-reflected multipath components of the modeled and measured CIRs. Since it is not feasible to create different modeled CIR sets $\mathcal{G}$ for many realizations of the surface waves and for different sea states, in this case it would be appropriate to skip the correlation-based depth/distance estimation that results in sets $\mathcal{M}^{(1)}(n)$. Instead, it would be possible to populate $\mathcal{M}^{(1)}(n)$ with all pairs of depth and distance values that satisfy RSSI bounds, and then proceed with the computation of the cross-correlations that lead to set $\mathcal{M}^{(2)}(n)$.

\section{F. Comparison against benchmark localization schemes}

We conclude our evaluation with a comparison among our algorithm, its preliminary version, ${ }^{1}$ and a benchmark scheme that, for every location index $n$ corresponding to a signal received by the buoy, chooses the most likely source location as the grid point in $\mathcal{M}^{(2)}(n)$ yielding the largest correlation value (dubbed "best point" in the following). This is akin to a classical fingerprint-based localization approach, where the fingerprint is defined as the value of Eq. (4). Figure 9 shows the CCDFs of the distance and bearing RMSE for all the above approaches carrying out all 


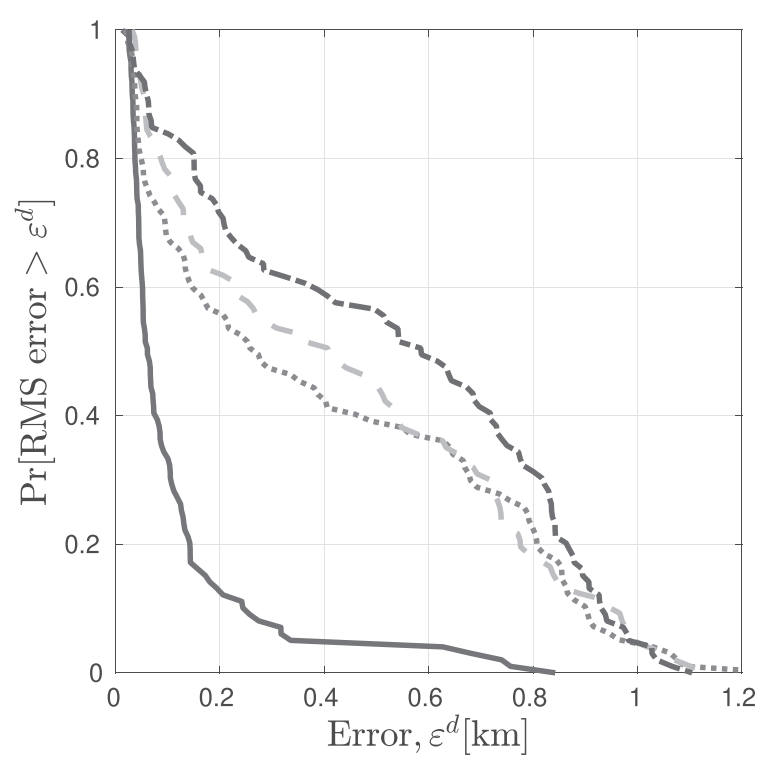

(a) CCDF of the distance RMSE

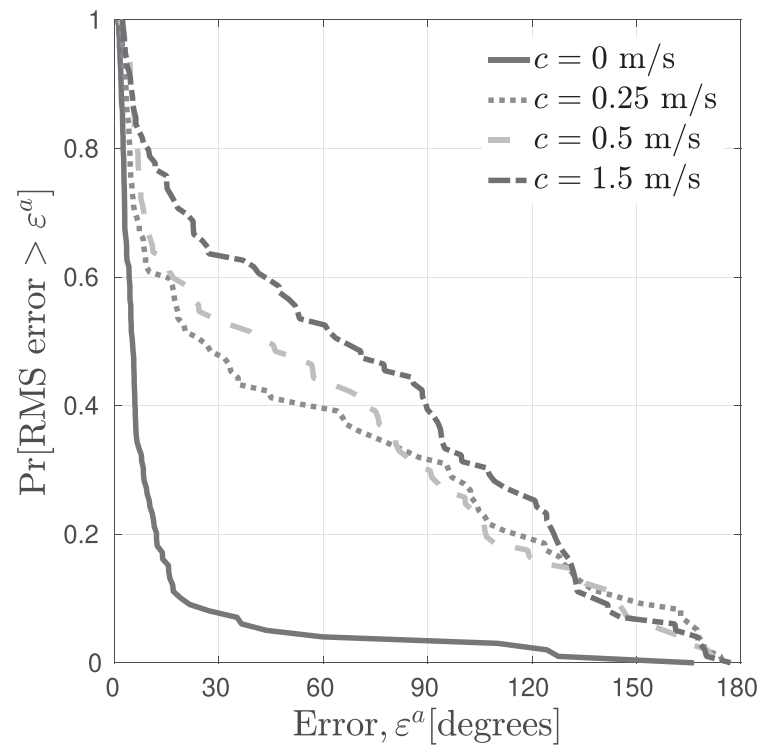

(b) CCDF of the bearing RMSE

FIG. 8. (Color online) Accuracy of the path estimation algorithm in the presence of imperfect SSP data. Increasing deviations from the actual SSP tend to significantly change the multipath arrival structure. For limited deviations, the median localization and angle error remain acceptable.

operations listed in Sec. III, including the forward- backward refinement of Sec. III D 3.

The results confirm the expectation that our approach achieves a lower estimation error. This is also due to the forward-backward search refinement, which reduces the chance that a comparatively low correlation value in the first point of the source's trajectory hampers the correct estimation of the whole path. Specifically, the median distance RMSE decreases from about 100 to about $50 \mathrm{~m}$, in the presence of a comparable angle RMSE. Although the best point scheme provides a good estimation of the bearing in these simulations, its distance error is still very significant, with $80 \%$ of the errors being greater than $100 \mathrm{~m}$. Moreover, while the tail

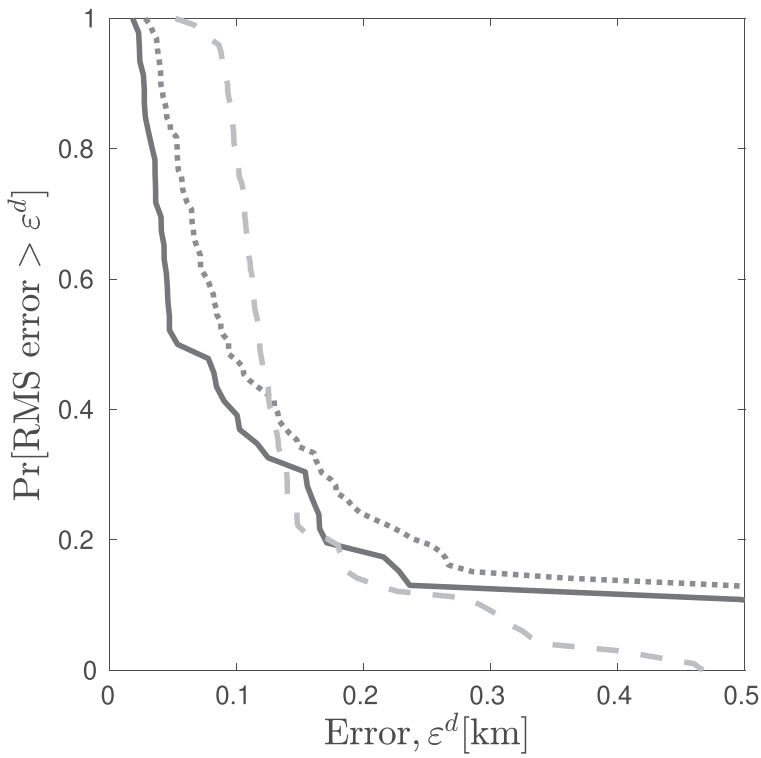

(a) CCDF of the distance RMSE of the error distribution for the best point scheme is better than for the algorithms in Ref. 1 and in this paper, these tails already correspond to significant errors (e.g., $>300 \mathrm{~m}$ in terms of distance and $>45^{\circ}$ in terms of bearing).

\section{EXPERIMENTAL RESULTS}

\section{A. Experiment setup}

In Sec. IV, we explored the performance of our localization scheme in simulations. Since these simulations rely heavily on a numerical acoustic propagation model, we now complete our analysis and show the performance achieved by our algorithm in a sea trial. The experiment was carried out in February 2017

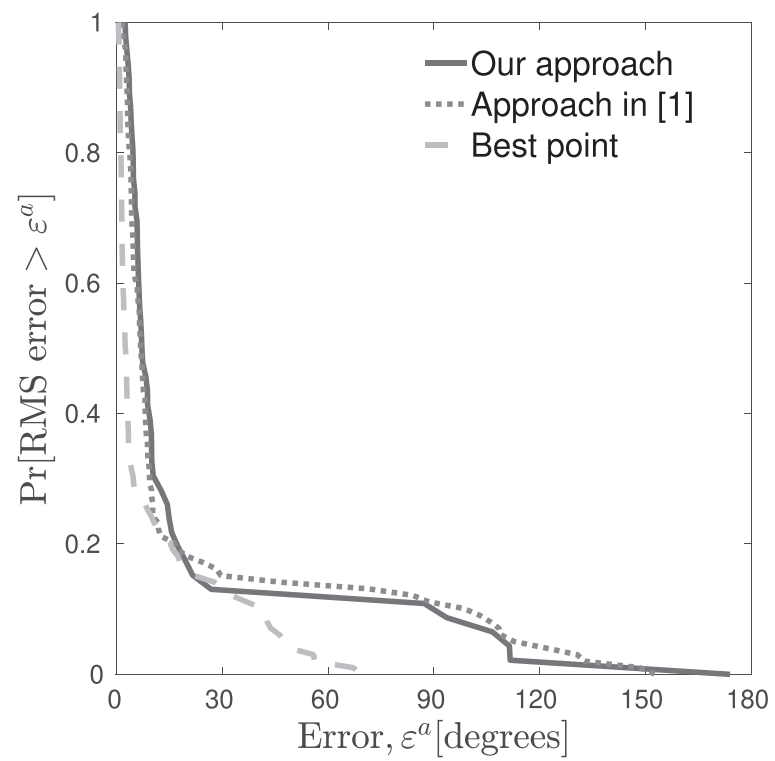

(b) CCDF of the bearing RMSE

FIG. 9. (Color online) Comparison among different location estimation schemes: our algorithm, the preliminary version of our approach in Ref. 1, and the best point benchmark (corresponding to selecting the location that yields the highest cross-correlation value). 


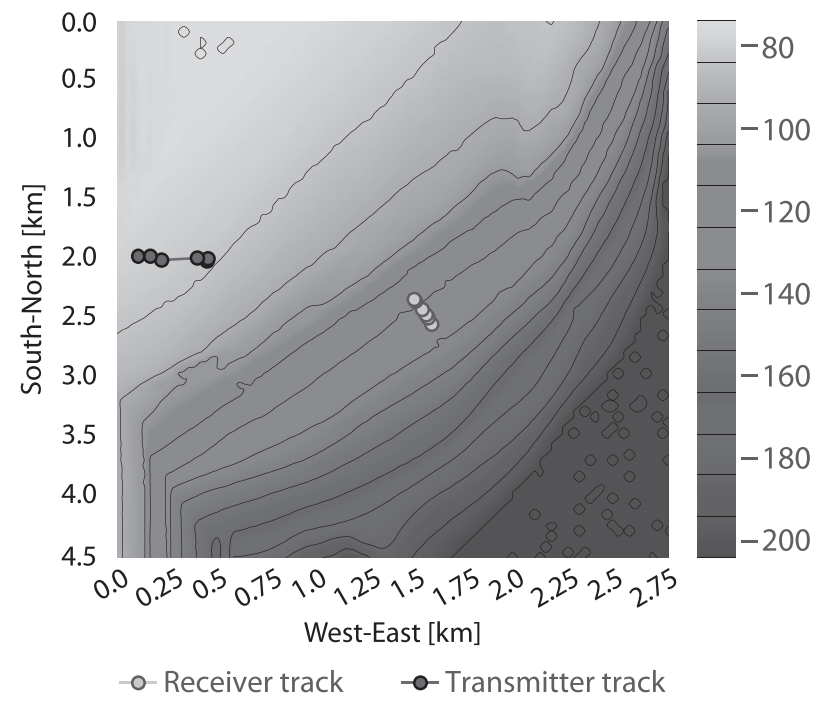

(a) Contour bathymetry map of the sea experiment area.

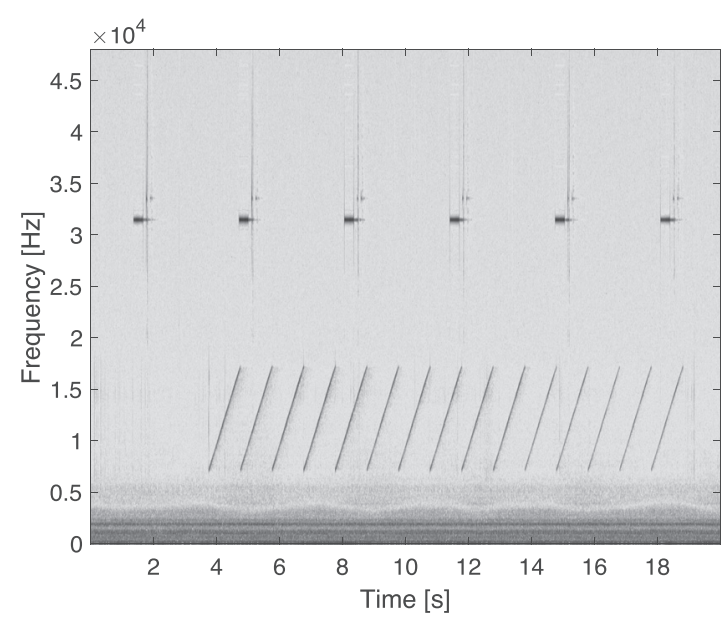

(b) Spectrogram of the received signals.

FIG. 10. (Color online) Setup of the sea experiment carried out in Mediterranean Sea waters near Haifa, Israel, in February 2017.

in northern Israel (coordinates $33^{\circ} 01^{\prime} 57.0^{\prime \prime} \mathrm{N} 34^{\circ} 55^{\prime} 41.2^{\prime \prime} \mathrm{E}$ ), in waters with a maximum depth of $140 \mathrm{~m}$. The measured sound speed was $1529 \mathrm{~m} / \mathrm{s}$ with a water temperature of $21^{\circ} \mathrm{C}$ at the sea surface, and $1521 \mathrm{~m} / \mathrm{s}$ with a water temperature of $17^{\circ} \mathrm{C}$ at the sea bottom. The sound speed gradient between the surface and bottom was approximately constant. The current at the water surface was roughly 0.5 knots, the wave height was roughly $40 \mathrm{~cm}$, and the sea bottom was sandy. A $5 \mathrm{~m}$ resolution bathymetry was collected using a Kongsberg EM 2040 $400 \mathrm{kHz}$ multibeam sonar. The bathymetry of the explored area is shown in Fig. 10(a), and included a steep slope ranging from 60 to $140 \mathrm{~m}$. The top-left side of the figure shows artificial data as no measurements were collected in that region.

The experiment included an $80 \mathrm{ft}$ long vessel, RV EDEN, and a $13 \mathrm{ft}$ rubber boat dragging a floating buoy from which an acoustic emitter was deployed, see Fig. 11. The rubber boat represented the opportunistic sounds source, and the RV EDEN represented the single receiver. During the transmissions, the distance between the vessels was roughly $1200 \mathrm{~m}$. The transmissions from the rubber boat included a sequence of 15 linear chirps at the frequency range of 7 to $17 \mathrm{kHz}$, each of duration of $1 \mathrm{~s}$. Transmissions were made with the EvoLogics S2C R $7 / 17 \mathrm{~W}$ underwater acoustic software defined modem at a source level of $170 \mathrm{~dB}$ re $1 \mu \mathrm{Pa} @ 1 \mathrm{~m}$ (EvoLogics, Berlin). Receptions at the RV EDEN were made through the custom uRadar recorder, whose receive sensitivity at the transmissions' frequency range is about $190 \mathrm{dBV}$ re $1 \mu \mathrm{Pa}$. Both the transmitter and the receiver were deployed at a depth of $10 \mathrm{~m}$. A timefrequency spectrogram of the received signals is shown in Fig. 10(b). Besides the transmitted chirp signals, we observe the signals of the RV EDEN's own echo-sounder. To mitigate the ambient noise as well as the signals of the echo-sounder, we filtered each chirp signal. Synchronization was performed using a normalized matched filter. ${ }^{42}$

\section{B. Results}

We start from Figs. 12 and 13, which detail the results of the comparison between the modeled CIRs and the measured one for nine consecutive received signals. The results are shown as a polar map centered around the location of the RV EDEN, where each contour line represents a distance of $300 \mathrm{~m}$ from the vessel. In Fig. 12, each small gray cross represents a possible position for the source, i.e., a comparison output that passed the detection threshold $\Theta_{D}$ (see Sec. III C) and was included in set $\mathcal{M}^{(2)}(i), i=1, \ldots, 9$. The thicker green cross marks the true location of the source. We observe that, for each of the nine received signals, many possible locations are obtained as a result of the crosscorrelation operations carried out by our method. Figure 14 shows one comparison between a CIR measured from a received signal and the CIR template constructed starting from Bellhop's output (light blue) and corresponding to a location close to the true location of the source. We observe that although the channel model is imperfect, all significant peaks in the measured CIR are well represented, leading to a good overall matching. However, other locations also lead to a similarly strong matching, resulting in several location

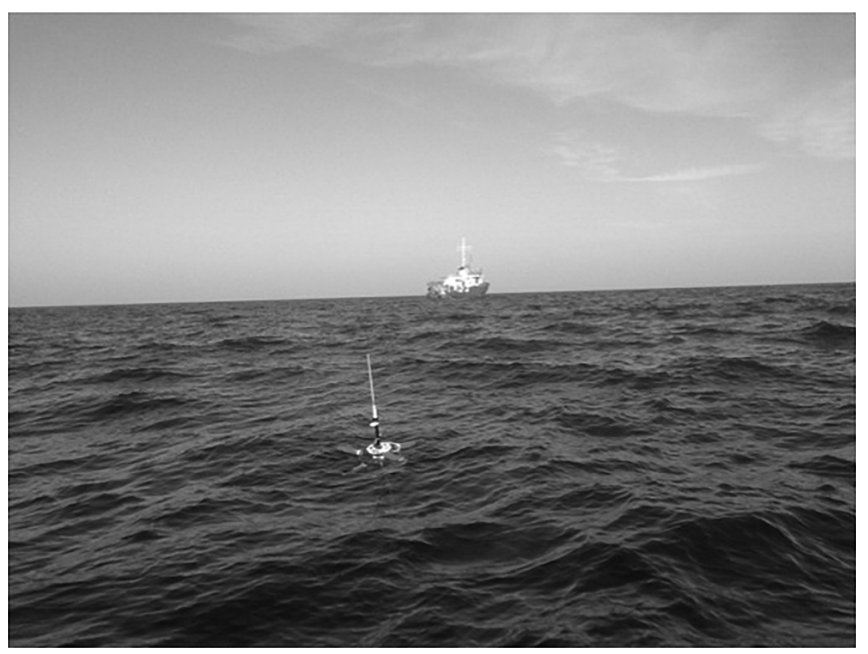

FIG. 11. (Color online) Picture of the buoy and ship from which the transmitter and receiver were deployed, respectively. 

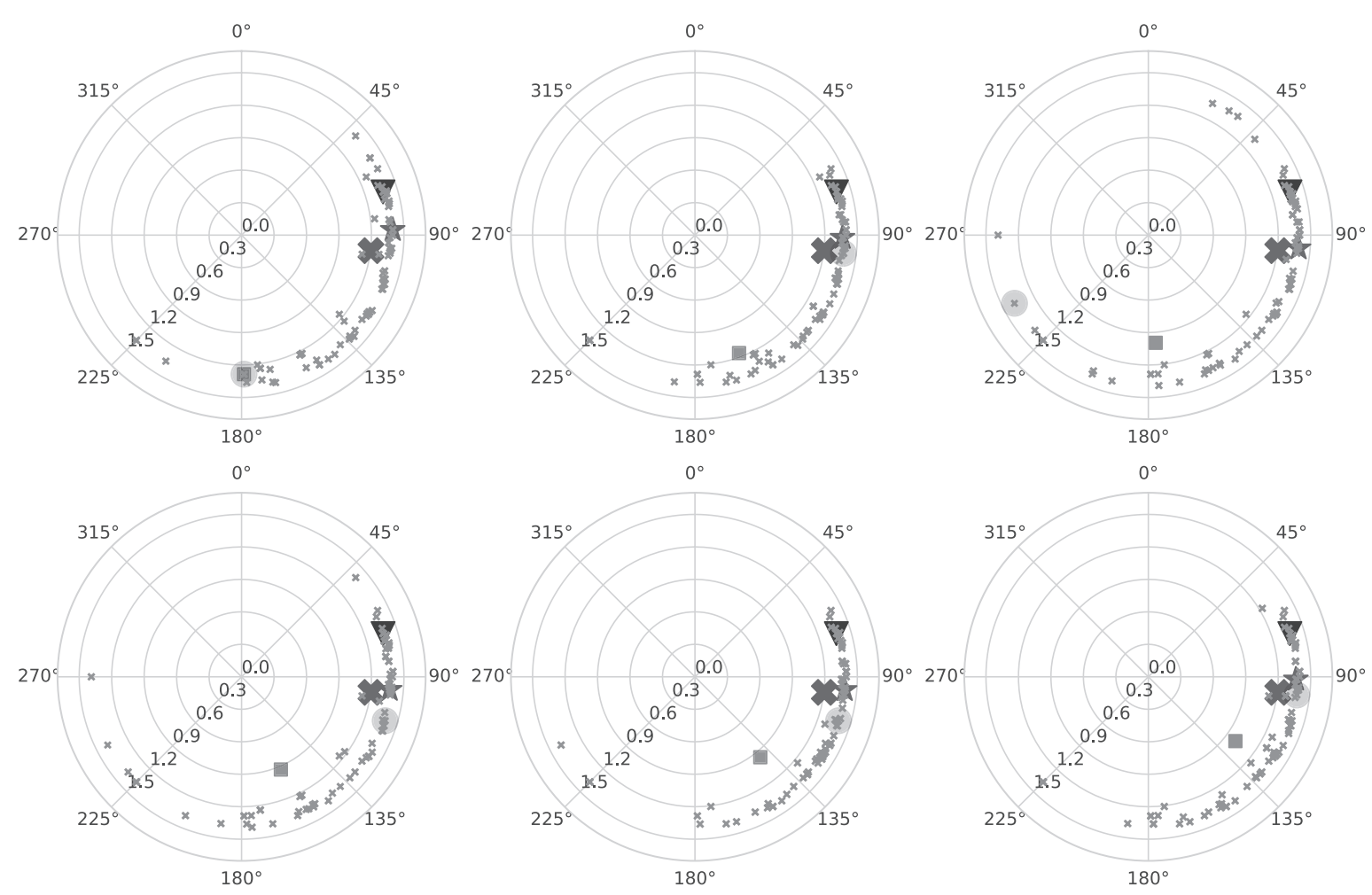

$0^{\circ}$
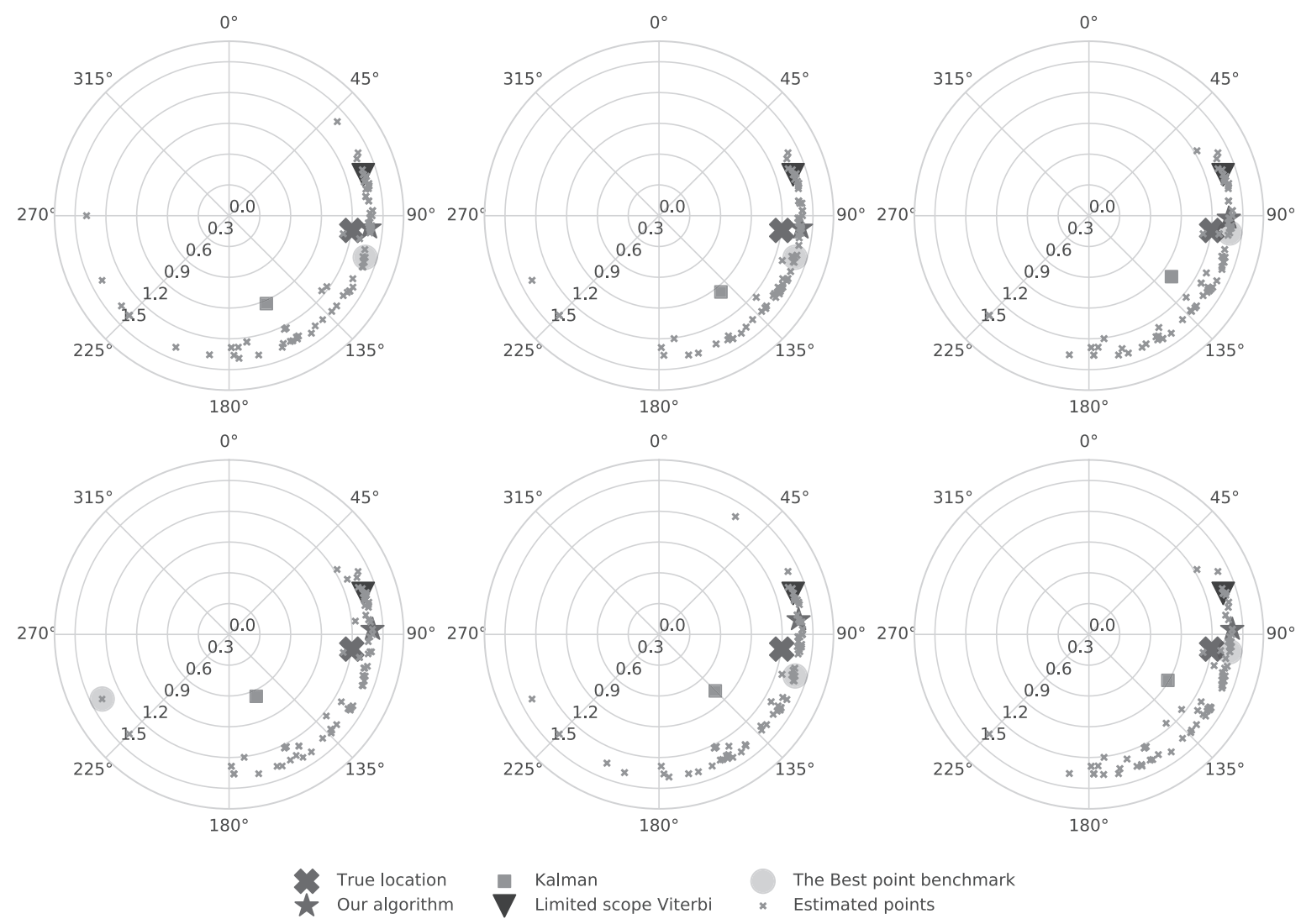

The Best point benchmark Estimated points

FIG. 12. (Color online) Sequence of location estimates for nine subsequent transmissions from a drifting source in the sea experiment, showing the true location of the source (green cross), the estimate of our algorithm (red triangles), the best point benchmark estimates (purple triangles), the Kalman filter results (gray squares), and the limited-scope Viterbi estimates (blue triangles).

estimates being significantly far from the source, and collectively resembling a random cloud of possible source locations (the small gray crosses). The best point algorithm (purple triangle), that points to the location yielding the maximum correlation for each signal, suffers from significant errors in three cases out of nine. These results support the simulation outcomes, showing that even when the bathymetry is fully known, relying only on the spatial diversity of the CIR yields significant residual uncertainty. Processing the outcome of the best point algorithm through a Kalman filter does not yield significantly better results, even if the filter is fed with the actual velocity of the AUV (in contrast with our approach, that only requires to know the maximum AUV velocity, $\left.v_{\max }^{\mathrm{s}}\right)$. The corresponding location estimates are shown in Fig. 14 as gray squares. Conversely, our algorithm (red triangles) exploits the trellis search to achieve a more precise estimation and removes outliers, resulting in a much smaller localization error.

We also compare the above results against those of the Viterbi algorithm. Given the size of the state space, in order to be able to run the algorithm we artificially reduce the search scope to a $90^{\circ}$ sector centered on the true bearing of the source, and to the distances ranging from 1000 to $1400 \mathrm{~m}$. While this gives a clear advantage to the Viterbi algorithm, it is a necessary step to allow the search space to fit in its data structures. The Viterbi results are shown as blue triangles in Fig. 12. We observe that the algorithm correctly predicts the fact that the source is static, but achieves a slightly worse location error despite the limitation of the search scope. This outcome is due to systematic, non-Gaussian errors incurred when modeling real underwater propagation using an acoustic propagation model under 


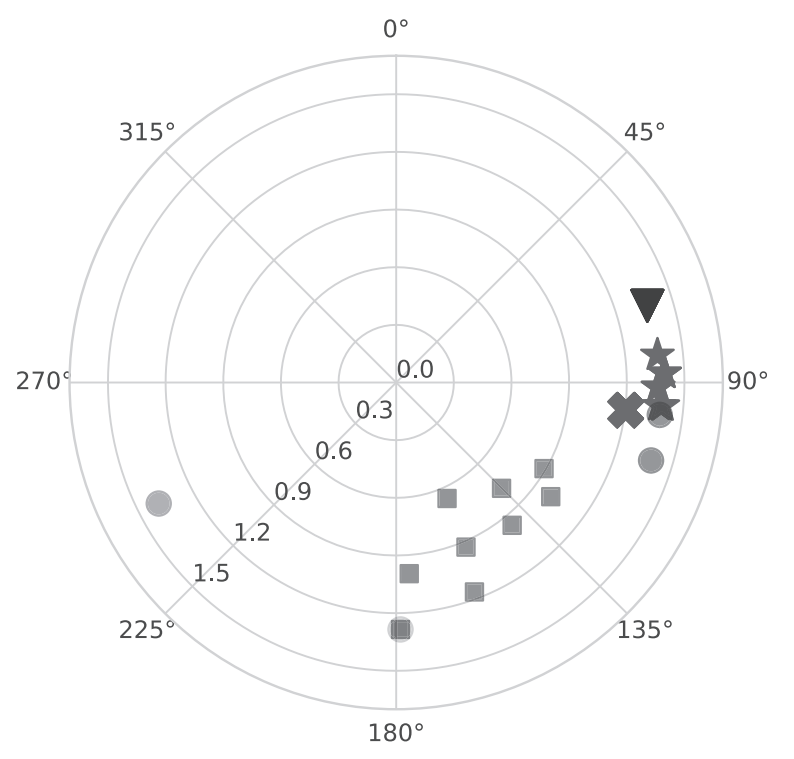

True location
Our algorithm
Kalman $\quad \begin{aligned} & \text { Limited scope Viterbi } \\ & \text { The Best point benchmark }\end{aligned}$

FIG. 13. (Color online) Final drifting source path estimate in the sea experiment, showing the true location of the source (green cross), the estimate of our algorithm (red triangles), the best point benchmark estimates (purple triangles), the Kalman filter results (gray squares), and the limited-scope Viterbi estimates (blue triangles). The total localization error for our algorithm is between $174 \mathrm{~m}$ $(5.8 \%)$ and $330 \mathrm{~m}(11 \%)$, with a bearing error between $2^{\circ}$ and $12^{\circ}$.

imperfect information (e.g., in this case, the resolution of the bathymetry and SSP data).

In Fig. 13, we summarize the path estimation results for all schemes with the same color coding as above. In this case, the green crosses represent the average location of the drifting RV EDEN throughout the experiment. The results show a nice match between the estimated location and the true one for our algorithm and the Viterbi algorithm, and for only a subset of the location estimates of the best point algorithm. The Kalman filter results also show that this method is very sensitive to incorrect estimates of the initial location of the AUV.

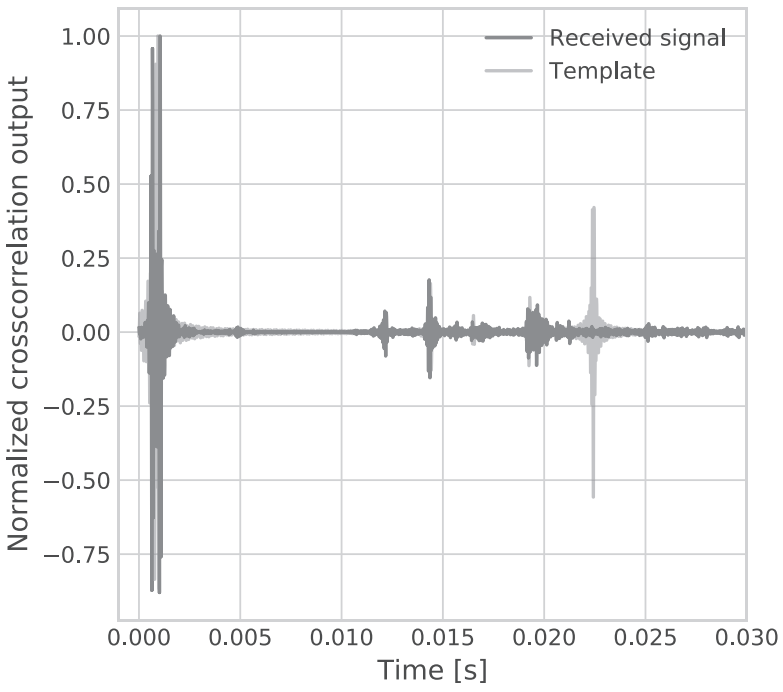

FIG. 14. (Color online) Comparison between a template CIR obtained from Bellhop (light blue) and the CIR measured from a signal received during the sea experiment.
The estimated locations predicted by our approach also correctly follow the drifting direction of the source boat. The total localization error for our algorithm is between 174 and $330 \mathrm{~m}$, with a bearing error between $2^{\circ}$ and $12^{\circ}$. While these errors may seem large, we argue that they are still acceptable for the task of localizing an AUV in a long term mission. This is because, after a few hours and especially in deep waters, the self-navigation system of the AUV would drift significantly: thus, any localization solution of limited expected error will benefit the operation. ${ }^{50}$ Moreover, compared to the typical detection range of roughly $5 \mathrm{~km}$ for the AUV's pinger (e.g., Ref. 51), the above reported localization error as in our experiment is still a good result. Given that this result was obtained using only one receiver in real sea conditions, it demonstrates well the applicability of our suggested localization method.

\section{CONCLUSIONS AND FUTURE WORK}

In this paper, we presented a novel approach for the acoustic localization of a non-cooperating AUV emitting acoustic signal. Our approach relies on a single passive and stationary receiving element and on the modeling of acoustic propagation given knowledge of the bathymetry, SSP, and bottom sediments in the deployment area. The method is based on the comparison of a CIR evaluated from a received acoustic signal, against a database of CIR fingerprints. As the latter are modeled instead of measured, we require no periodic channel fingerprint acquisition in the area around the receiver. To filter noise, locations that yield a good match between the measured and the modeled channels are arranged into a trellis. A location path is then estimated while limiting transitions between the trellis nodes according to an assumed maximum velocity for the AUV. Our approach makes it possible to estimate the path traveled by the AUV with low complexity and with high accuracy. Such accuracy decreases (but still remains sufficient for a variety of applications) if the receiver holds outdated environmental data. A proof-of-concept sea experiment demonstrates the applicability of our method to real sea conditions with a localization error as low as $5.8 \%$, which is a remarkably good accuracy given the use of a single stationary receiver and the realistic imperfect bathymetry and SSP measurements.

Future work will include a refinement considering a finer non-uniform grid in locations where the input data shows the largest variability, as well as extending the localization to multiple targets.

\section{ACKNOWLEDGMENTS}

This work was sponsored in part by the European Commission's Horizon 2020 research and innovation programme under grant agreement No. 773753 (SYMBIOSIS), by the Israeli Ministry of Science and Technology (Grant No. 3-12473) and by Germany's Federal Ministry of Education and Research (BMBF) (Grant No. 3-15249).

${ }^{1}$ E. Dubrovinskaya, R. Diamant, and P. Casari, "Anchorless underwater acoustic localization," in Proceedings of the IEEE, 14th Workshop on Positioning, Navigation and Communications (2017).

${ }^{2}$ J. J. Leonard and A. Bahr, "Autonomous underwater vehicle navigation," in Handbook of Ocean Engineering (Springer, New York, 2016), pp. 341-358. 
${ }^{3}$ Z. Zhou, Z. Peng, J.-H. Cui, Z. Shi, and A. Bagtzoglou, "Scalable localization with mobility prediction for underwater sensor networks," IEEE Trans. Mobile Comput. 10(3), 335-348 (2011).

${ }^{4}$ R. Diamant and L. Lampe, "Underwater localization with timesynchronization and propagation speed uncertainties," IEEE Trans. Mobile Comput. 12(7), 1257-1269 (2013).

${ }^{5}$ K.-C. Lee, J.-S. Ou, M.-C. Huang, and M.-C. Fang, "A novel location estimation based on pattern matching algorithm in underwater environments," Applied Acoust. 70(3), 479-483 (2009).

${ }^{6}$ H.-P. Tan, R. Diamant, W. K. Seah, and M. Waldmeyer, "A survey of techniques and challenges in underwater localization," Ocean Eng. 38(14), 1663-1676 (2011).

${ }^{7}$ L. Paull, S. Saeedi, M. Seto, and H. Li, "AUV navigation and localization: A review,” IEEE J. Ocean. Eng. 39(1), 131-149 (2014).

${ }^{8}$ J. Reis, M. Morgado, P. Batista, P. Oliveira, and C. Silvestre, "Design and experimental validation of a USBL underwater acoustic positioning system," Sensors 16(9), 1-23 (2016).

${ }^{9}$ M. Erol-Kantarci, H. Mouftah, and S. Oktug, "Localization techniques for underwater acoustic sensor networks," IEEE Commun. Mag. 48(12), 152-158 (2010).

${ }^{10}$ S. Gezici, "A survey on wireless position estimation," Wireless Pers. Commun. 44(3), 263-282 (2008).

${ }^{11}$ Z. Zhang, C. L. Law, and Y. L. Guan, "Modified phase-only correlator with kurtosis-based amplified-noise suppression," IEEE Trans. Wireless Commun. 9(11), 3341-3345 (2010).

${ }^{12} \mathrm{~L}$. Mu, G. Kuo, and N. Tao, "A novel ToA location algorithm using LOS range estimation for NLOS environments," in Proceedings of the IEEE Transactions on Vehicular Technology, Melbourne, Australia (2006).

${ }^{13}$ J. Ash and R. Moses, "Acoustic time delay estimation and sensor network self-localization: Experimental results,” J. Acoust. Soc. Am. 118(2), 841-850 (2005).

${ }^{14}$ D. McCrady, L. Doyle, H. Forstrom, T. Dempsey, and M. Martorana, "Mobile ranging using low-accuracy clocks," IEEE Trans. Microwave Theory Tech. 48(6), 951-957 (2000).

${ }^{15}$ S. Fischer, H. Grubeck, A. Kangas, H. Koorapaty, E. Larsson, and P. Lundqvist, "Time of arrival estimation of narrowband TDMA signals for mobile positioning," in IEEE International Symposium on Personal, Indoor and Mobile Radio Communications (PIMRC) (1998), pp. 451-455.

${ }^{16}$ E. Dubrovinskaya, I. Nissen, and P. Casari, "On the accuracy of passive multipath-aided underwater range estimation," in IEEE Third Underwater Communications and Networking Conference (UComms), Lerici, Italy (2016).

${ }^{17}$ C. O. Tiemann, M. B. Porter, and L. N. Frazer, "Localization of marine mammals near Hawaii using an acoustic propagation model," J. Acoust. Soc. Am. 115(6), 2834-2843 (2004).

${ }^{18}$ J. Gebbie, M. Siderius, and J. S. Allen III, "A two-hydrophone range and bearing localization algorithm with performance analysis," J. Acoust. Soc. Am. 137(3), 1586-1597 (2015).

${ }^{19}$ A. B. Baggeroer, W. A. Kuperman, and P. N. Mikhalevsky, "An overview of matched field methods in ocean acoustics," IEEE J. Ocean. Eng. 18(4), 401-424 (1993).

${ }^{20}$ J. S. Perkins and W. Kuperman, "Environmental signal processing: Threedimensional matched-field processing with a vertical array," J. Acoust. Soc. Am. 87(4), 1553-1556 (1990).

${ }^{21}$ K. L. Gemba, W. S. Hodgkiss, and P. Gerstoft, "Adaptive and compressive matched field processing," J. Acoust. Soc. Am. 141(1), 92-103 (2017).

${ }^{22} \mathrm{~S}$. Shakeri and G. Leus, "Underwater ultra-wideband fingerprinting-based sparse localization," in IEEE Workshop on Signal Processing Advances in Wireless Communications (SPAWC), Toronto, Canada (2014).

${ }^{23} \mathrm{~S}$. Rakotonarivo and W. Kuperman, "Model-independent range localization of a moving source in shallow water," J. Acoust. Soc. Am. 132(4), 2218-2223 (2012).

${ }^{24}$ C. M. Verlinden, J. Sarkar, B. D. Cornuelle, and W. A. Kuperman, "Determination of acoustic waveguide invariant using ships as sources of opportunity in a shallow water marine environment," J. Acoust. Soc. Am. 141(2), EL102-EL107 (2017).

${ }^{25}$ L. Liao, Y. V. Zakharov, and P. D. Mitchell, "Underwater localization based on grid computation and its application to transmit beamforming in multiuser UWA communications," IEEE Access 6, 4297-4307 (2018).

${ }^{26}$ M. S. I. Seddik, L. Jaulin, and J. Grimsdale, "Phase based localization for underwater vehicles using interval analysis," Math. Comp. Sci. 8(3), 495-502 (2014).

${ }^{27}$ H. Liu, H. Darabi, P. Banerjee, and J. Liu, "Survey of wireless indoor positioning techniques and systems," IEEE Trans. Syst., Man, Cybern. C 37(6), 1067-1080 (2007).
${ }^{28} \mathrm{~K}$. Kaemarungsi and P. Krishnamurthy, "Modeling of indoor positioning systems based on location fingerprinting," in IEEE Annual Joint Conference INFOCOM, Computer and Communications Societies, Hong Kong, China (2004), Vol. 2, pp. 1012-1022.

${ }^{29}$ Y. Jin, W. Soh, and W. Wong, "Indoor localization with channel impulse response based fingerprint and nonparametric regression," IEEE Trans. Wireless Commun. 9(3), 1120-1127 (2010).

${ }^{30} \mathrm{X}$. Guo and N. Ansari, "Localization by fusing a group of fingerprints via multiple antennas in indoor environment," IEEE Trans. Veh. Technol. 66(11), 9904-9915 (2017).

${ }^{31}$ P. Tseng, Y. Chan, Y. Lin, D. Lin, N. Wu, and T. Wang, "Ray-tracingassisted fingerprinting based on channel impulse response measurement for indoor positioning," IEEE Trans. Instrum. Meas. 66(5), 1032-1045 (2017).

${ }^{32} \mathrm{~N}$. Etemadyrad, "A sequential detection approach to indoor positioning using RSS-based fingerprinting," Master's thesis, George Mason University (2017).

${ }^{33}$ P. K. Yadav and W. Meng, "Mobile targets localization in a field area using moving Gaussian peaks and probability map," in Proceedings of the 11th IEEE International Conference on Control \& Automation (ICCA), Taichung, Taiwan (2014).

${ }^{34}$ Z. Xiao, H. Wen, A. Markham, and N. Trigoni, "Lightweight map matching for indoor localisation using conditional random fields," in Proceedings of the 13th International Symposium on Information Processing in Sensor Networks, Berlin, Germany (2014).

${ }^{35}$ Z. Wei, Y. Zhao, X. Liu, and Z. Feng, "DoA-LF: A location fingerprint positioning algorithm with millimeter-wave," IEEE Access 5, 22678-22688 (2017).

${ }^{36}$ J. Palacios, G. Bielsa, P. Casari, and J. Widmer, "Single- and multipleaccess point indoor localization for millimeter-wave networks," IEEE Trans. Wireless Commun. 18(3), 1927-1942 (2019).

${ }^{37}$ F. Jensen, W. Kuperman, M. Porter, and H. Schmidt, Computational Ocean Acoustics, 2nd ed. (Springer-Verlag, New York, 1984).

${ }^{38}$ HLS Research, "Bellhop code," http://oalib.hlsresearch.com/Rays/ index.html (Last viewed May 2018).

${ }^{39} \mathrm{P}$. Qarabaqi and M. Stojanovic, "Statistical characterization and computationally efficient modeling of a class of underwater acoustic communication channels," IEEE J. Ocean. Eng. 38(4), 701-717 (2013).

${ }^{40}$ L. M. Wolff, E. Szczepanski, and S. Badri-Hoeher, "Acoustic underwater channel and network simulator," in Proceedings of IEEE OCEANS, Yeosu, Korea (2012), pp. 1-6.

${ }^{41}$ Note that this information is part of Bellhop's standard output data, and that we do not need to track any propagation history analysis for any of the components of the measured CIR.

${ }^{42} \mathrm{R}$. Diamant, "Closed form analysis of the normalized matched filter with a test case for detection of underwater acoustic signals," IEEE Access 4, $8225-8235$ (2016).

${ }^{43}$ R. Diamant, H.-P. Tan, and L. Lampe, "LOS and NLOS classification for underwater acoustic localization," IEEE Trans. Mobile Comput. 13(2), 311-323 (2014).

${ }^{44} \mathrm{Q}$. Wang, L. Wei, and R. Kennedy, "Iterative Viterbi decoding, trellis shaping, and multilevel structure for high-rate parity-concatenated TCM," IEEE Trans. Commun. 50(1), 48-55 (2002).

${ }^{45} \mathrm{~L}$. Marchetti and R. Reggiannini, "An efficient receiver structure for sweep-spread-carrier underwater acoustic links," IEEE J. Ocean. Eng. 41(2), 440-449 (2016).

${ }^{46}$ C. Boya, M. Ruiz-Llata, J. Posada, A. Garcia-Souto, and A. José, "Identification of multiple partial discharge sources using acoustic emission technique and blind source separation," IEEE Trans. Dielectr. Electr. Insul. 22(3), 1663-1673 (2015).

${ }^{47}$ S. Byun, S. Kim, C. Park, K. Kim, and C. Lee, "Cyclostationary analysis of underwater noise for vehicle propeller monitoring," in Proceedings of the MTS/IEEE OCEANS, Monterey, CA (2016).

${ }^{48}$ D. Divins and D. Metzger, "US coastal relief model," (2016), http:// www.ngdc.noaa.gov/mgg/coastal/coastal.html (Last viewed November 30, 2019).

${ }^{49}$ R. Diamant, A. Knap, S. Dahan, I. Mardix, J. Walpert, and S. DiMarco, "THEMO: The Texas A\&M-University of Haifa-Eastern Mediterranean Observatory," in Proceedings of MTS/IEEE OCEANS, Kobe, Japan (2018).

${ }^{50} \mathrm{~T}$. Alexandri and R. Diamant, "A reverse bearings only target motion analysis for autonomous underwater vehicle navigation,” IEEE Trans. Mobile Comput. 18(3), 494-506 (2019).

${ }^{51}$ Information on low-frequency pingers available at http://www. jwfishers.com/products/pingers-low.html (Last viewed November 30, 2019). 